\title{
Regularity and blow up in a surface growth model
}

\author{
Dirk Blömker and Marco Romito
}

Communicated by James Robinson, received April 8, 2009.

\begin{abstract}
The paper contains several regularity results and blow-up criteria for a surface growth model, which seems to have similar properties to the 3D Navier-Stokes, although it is a scalar equation. As a starting point we focus on energy methods and Lyapunov-functionals.
\end{abstract}

\section{Contents}

1. Introduction 227

2. Existence and uniqueness in a critical space 230

3. Regularity 240

4. Blow up 243

Appendix A. An inequality for the non-linearity 248

Appendix B. Blow up for ODEs 250

Appendix C. Analytic Semigroups 251

Acknowledgements $\quad 251$

$\begin{array}{ll}\text { References } & 251\end{array}$

\section{Introduction}

Throughout this paper we analyse a PDE originated by a model of surface growth. Our main motivation is to carry over the program developed for 3DNavier-Stokes to this equation, in order to study the possible blow up of solutions. This paper is the starting point focusing mainly on Hilbert space theory (although the understanding of the Navier-Stokes equation has gone much further, see [13] for results that are the actual state of the art).

2000 Mathematics Subject Classification. 35B33, 35B45, 35B65, 35K55, 35Qxx, 60H15.

Key words and phrases. surface growth, critical space, uniqueness, regularity, blow up, Leray estimates, Lyapunov function.

The second author gratefully acknowledges the support of Hausdorff Research Institute for Mathematics (Bonn), through the Junior Trimester Program on Computational Mathematics, and the hospitality of Augsburg Universität. 
Details on the model can be found in Raible et al. [19], [20] or Siegert and Plischke [24]. In its simplest version, it is given by

$$
\partial_{t} h=-\partial_{x}^{4} h-\partial_{x}^{2}\left(\partial_{x} h\right)^{2}
$$

subject to periodic boundary conditions on $[0, L]$ and $\int_{0}^{L} h d x=0$. Although the surface is not periodic, these boundary conditions together with the assumption of a moving frame are the standard conditions in models of this type. Sometimes the model has been considered also on the whole real line without decay condition at infinity, even though we do not examine this case here.

From a mathematical point of view Neumann or Dirichlet boundary conditions are quite similar for the problem studied here. The key point ensured by any of these boundary conditions is that there is a suitable cancellation in the non-linearity, namely

$$
\int_{0}^{L} h\left(h_{x}^{2}\right)_{x x} d x=0
$$

which is the main (and probably only) ingredient to derive useful a-priori estimates.

The main terms in the equation are the dominant linear operator, and the quadratic non-linearity. Sometimes the equation is considered with a linear instability $-h_{x x}$, which leads to the formation of hills, and the Kuramoto-Sivashinsky-type nonlinearity $\left(h_{x}\right)^{2}$ leading to a saturation in the coarsening of hills. Both terms are neglected here. They are lower order terms not important for questions regarding regularity and blow up. Moreover, the presence of these terms complicates calculations significantly (see [5]).

Furthermore, the equation is usually perturbed by space-time white noise (see for instance [7]), which we also neglect here, although many results do hold for the stochastic PDE also.

For general surveys on surface growth processes and molecular beam epitaxy see Barabási and Stanley [1] or Halpin-Healy and Zhang [12].

1.1. Existence of solutions. There are two standard ways of treating the existence of solutions. The first one relies on the spectral Galerkin method and uses energy type estimates for the approximation, which by some compactness arguments ensure the convergence of a subsequence. See [25], or for the stochastically perturbed equation $[4,3,7]$. In all cases initial conditions in $L^{2}$ ensure the existence, but not uniqueness, of global solutions.

The second way uses fixed point arguments to show local uniqueness and regularity using the mild formulation. An application of this method is given in [6], although the optimal case is not treated. In Section 2 we give a local existence result, which is optimal in the sense that initial conditions are in a critical space (see [10] for a detailed account on this topic for the Navier-Stokes equations). We also establish uniqueness among mild solutions and, less trivially, among weak solutions. For these smooth local solutions we can easily show energy estimates, and discuss possible singularities and blow up.

Standard arguments assure uniqueness of global solutions using a fixed point argument in $C^{0}\left([0, T], H^{1}\right)$ for sufficiently small regular data in $H^{1}$. We can even go below that for uniqueness of solutions in $H^{\alpha}$ for any $\alpha \geq \frac{1}{2}$. This improves results of [6]. But we are still not able to prove uniqueness of global solutions without a smallness condition on the initial data. Nevertheless, we can give easily several 
conditions that imply uniqueness of global solutions. All of them assume regularity in critical spaces or more regularity (see Section 3).

In Section 4 we study possible singularities and blow up. Based on energy-type estimates, we establish Leray type estimates for lower bounds on blow up in terms of $H^{\alpha}$-norms. Moreover, we study an upper bound on the Hausdorff-dimension of the set of singularities in time, and show that a blow up to $-\infty$ is more likely to happen.

REMARK 1.1. All results for regularity and Leray type estimates are based on energy estimates. These are optimal in the sense that they also hold for complex valued solutions. Furthermore, using the ideas of [15], [16], one should be able to construct a complex valued solution with strictly positive Fourier coefficients that actually blows up in finite time. This is the subject of a work in progress.

This would show that results based on energy-estimates are useful to describe a possible blow up, but they alone will never be able to rule it out.

1.2. The energy inequality. We outline the standard idea for energy estimates, which is to our knowledge the only useful idea for this equation. If we formally multiply the equation by $h$ and integrate with respect to $x$, then we obtain using (1.2),

$$
|h(t)|_{L^{2}}^{2}+2 \int_{0}^{t}\left|\partial_{x}^{2} h(s)\right|_{L^{2}}^{2} d s \leq|h(0)|_{L^{2}}^{2} .
$$

Thus, using the Poincare inequality,

$$
|h(t)|_{L^{2}} \leq \mathrm{e}^{-c t}|h(0)|_{L^{2}}^{2} \quad \text { and } \quad \int_{0}^{\infty}|h(t)|_{H^{2}}^{2} d t \leq|h(0)|_{L^{2}}^{2} .
$$

As explained before this estimate is only valid for smooth local solutions, or one could use spectral Galerkin approximation to verify it for global solutions. Note that the regularity implied by this estimate is enough for proving existence of solutions, but is lower than the critical regularity, that is, the minimal amount of regularity that would ensure uniqueness.

1.3. A Lyapunov-type functional. We can prove another a-priori estimate either for smooth local solutions or via spectral Galerkin approximations,

$$
\begin{aligned}
\frac{1}{\alpha^{2}} \frac{d}{d t} \int_{0}^{L} \mathrm{e}^{\alpha h} d x & =\int_{0}^{L} \mathrm{e}^{\alpha h} h_{x} h_{x x x} d x+2 \int_{0}^{L} \mathrm{e}^{\alpha h} h_{x}^{2} h_{x x} d x \\
& =-\int_{0}^{L} \mathrm{e}^{\alpha h} h_{x x}^{2} d x+(2-\alpha) \int_{0}^{L} \mathrm{e}^{\alpha h} h_{x}^{2} h_{x x} d x \\
& =-\int_{0}^{L} \mathrm{e}^{\alpha h} h_{x x}^{2} d x-\frac{1}{3}(2-\alpha) \alpha \int_{0}^{L} \mathrm{e}^{\alpha h} h_{x}^{4} d x .
\end{aligned}
$$

Thus, for $\alpha \in(0,2)$,

$$
\int_{0}^{L} \mathrm{e}^{\alpha h(t)} d x \leq \int_{0}^{L} \mathrm{e}^{\alpha h(0)} d x \text { for all } t>0
$$

and

$$
\frac{(2-\alpha)}{3} \alpha^{3} \int_{0}^{\infty} \int_{0}^{L} \mathrm{e}^{\alpha h} h_{x}^{4} d x d t+\alpha^{2} \int_{0}^{\infty} \int_{0}^{L} \mathrm{e}^{\alpha h} h_{x x}^{2} d x d t \leq \int_{0}^{L} \mathrm{e}^{\alpha h(0)} d x .
$$




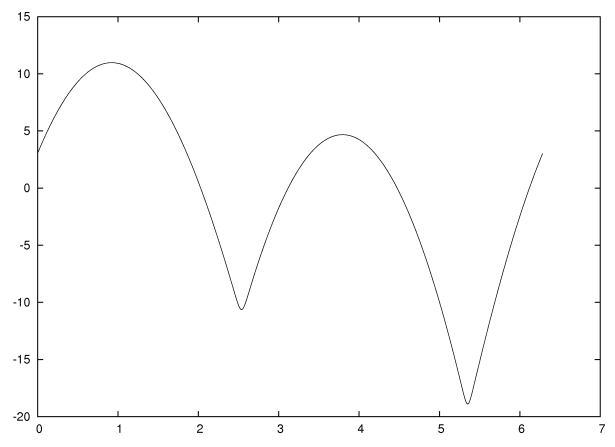

Figure 1. A snapshot of a numerical solution to the surface growth equation with additional linear instability $-30 h_{x x}$. The hills look like parabolas with sharp valleys in between.

With some more effort (see Stein and Winkler [25]) one can see that these terms are bounded independently of $h(0)$ for large $t$.

The positive part $h^{+}=\max \{0, h\}$ now has much more regularity than the negative part $h^{-}=\max \{0, h\}$, so a possible blow up seems to be more likely to $-\infty$ than to $+\infty$. We will illustrate this in Subsection 4.3. Unfortunately, this regularity is still not sufficient for uniqueness of solutions.

\section{Existence and uniqueness in a critical space}

Prior to the details on some regularity criteria for equation (1.1), we introduce the scaling heuristic which explains the formulae that relate the different exponents in the results of the paper. An account on the scaling heuristic for the Navier-Stokes equations can be found for example in Cannone [10], such argument are the basis of the celebrated result on partial regularity for Navier-Stokes of Caffarelli, Kohn, and Nirenberg [8].

The rationale behind the method is the following. First, notice that the equations are invariant for the scaling transformation

$$
h(t, x) \longrightarrow h_{\lambda}(t, x)=h\left(\lambda^{4} t, \lambda x\right) .
$$

If $X$ is a functional space for $h$ (for example $L^{\infty}\left(0, T ; L^{2}(0, L)\right)$ ), we can consider how the norm of $X$ scales with respect to the transformation (2.1) above. Say the following relation holds,

$$
\left\|h_{\lambda}\right\|_{X}=\lambda^{-\alpha}\|h\|_{X}
$$

We have the three cases

1. sub-critical case for $\alpha<0$,

2. critical case for $\alpha=0$,

3. super-critical case for $\alpha>0$.

The super-critical case corresponds to small-scales behaviour and is related to low regularity, typically to topologies where possibly existence can be proved, but no regularity or uniqueness. For example, one gets $\alpha=\frac{1}{2}$ (hence, super-critical) for $X=L^{\infty}\left(0, \infty ; L^{2}\right)$ or $X=L^{2}\left(0, T ; \dot{H}^{2}\right)$, which are the spaces where existence of global weak solutions can be proved. 
The general scheme is the following. Consider spaces $X$ (depending on the space variable) and $Y_{T}$ (depending on both variables, with $t$ up to $T>0$ ), then in order to have a regularity criterion based on $Y_{T}$, the following statements must hold,

1. there is a unique local solution for every initial condition in $X$,

2. the unique local solution we have found is regular, and

3. the unique local solution can be continued up to time $T$, as long as its norm in $Y_{T}$ stays bounded.

The above analysis has been extensively carried out by a large number of authors for the three dimensional Navier-Stokes equations (see for examples references in Cannone [10]). The first paper dealing with such aims were Prodi [18] and Serrin [23], see also Beale, Kato and Majda [2].

2.0.1. Function spaces. We shall mainly work in the hierarchy of Sobolev spaces of Hilbert type. Since the equations are considered on $[0, L]$ with periodic boundary conditions and zero space average, we shall use the following homogeneous fractional Sobolev spaces. For $\alpha>0$,

$$
\dot{H}^{\alpha}=\left\{u \in L^{2}(0, L): u(\cdot+L)=u(\cdot), \quad u_{0}=0, \quad \sum_{k \neq 0} k^{2 \alpha}\left|u_{k}\right|^{2}<\infty\right\},
$$

where $u_{k}$ is the $k^{\text {th }}$ Fourier coefficient, and $\dot{H}^{-\alpha}=\left(\dot{H}^{\alpha}\right)^{\prime}$. We shall consider the norm on $\dot{H}^{\alpha}$ defined by

$$
|u|_{\alpha}^{2}=\sum_{k \neq 0} k^{2 \alpha}\left|u_{k}\right|^{2}
$$

which is equivalent to the norm of the Sobolev space $H^{\alpha}(0, L)$ on $\dot{H}^{\alpha}$.

We also use the space $L^{p}$ with norm $|\cdot|_{L^{p}}$ for the Lebesgue space of functions with integrable $p$-th power, the space $W^{k, p}$ with norm $|\cdot|_{W^{k, p}}$ for the Sobolev space, where the $k$-th derivative is in $L^{p}$, and the space $C^{k}$ of $k$-time continuously differentiable functions with the supremum-norm.

2.1. Existence and uniqueness in $\dot{H}^{\frac{1}{2}}$. This section is devoted to the proof of existence and uniqueness in the critical space $\dot{H}^{\frac{1}{2}}$, which improves significantly some results of Blömker and Gugg [6]. Here we shall follow the results of Fujita and Kato [11] on the Navier-Stokes equations with initial conditions in the critical Sobolev Hilbert space. This is optimal in the sense that local existence and uniqueness with lower regularity should imply uniqueness by rescaling.

Definition 2.1. Given $T>0, \delta>0$ and $\alpha \in\left(0, \frac{1}{2}\right)$, define the complete metric space $\mathcal{S}_{\alpha}=\mathcal{S}_{\alpha}(T)$ as

$$
\mathcal{S}_{\alpha}(T)=\left\{u \in C\left((0, T] ; \dot{H}^{1+\alpha}\right): \sup _{s \in(0, T]}\left\{s^{\frac{2 \alpha+1}{8}}|u(s)|_{1+\alpha}\right\}<\infty\right\},
$$

with norm

$$
\|u\|_{\alpha, T}=\sup _{s \in(0, T]}\left\{s^{\frac{2 \alpha+1}{8}}|u(s)|_{1+\alpha}\right\}
$$

and the $\delta$-ball

$$
\mathcal{S}_{\alpha}^{\delta}(T)=\left\{u \in \mathcal{S}_{\alpha}(T):\|u\|_{\alpha, T} \leq \delta\right\} .
$$

Let us remark that for any $h \in \mathcal{S}_{\alpha}(T), \widetilde{\alpha} \in(0, \alpha)$ and $\delta>0$ one can find $\widetilde{T} \in(0, T)$ such that $h \in \mathcal{S}_{\widetilde{\alpha}}^{\delta}(\widetilde{T})$. 
THEOREM 2.2. Given an arbitrary initial condition $h_{0} \in \dot{H}^{\frac{1}{2}}$, there exists a time $T_{\bullet}>0$, depending only on $h_{0}$, such that there is a unique solution $h \in$ $C\left(\left[0, T_{\bullet}\right) ; \dot{H}^{\frac{1}{2}}\right)$ to problem (1.1). Moreover,

1. $h \in C^{\infty}\left(\left(0, T_{\bullet}\right) \times[0, L]\right)$,

2. the solution satisfies the energy equality

$$
|h(t)|_{L^{2}}^{2}+2 \int_{0}^{t}\left|h_{x x}\right|_{L^{2}}^{2} d s=|h(0)|_{L^{2}}^{2},
$$

for all $t<T_{\bullet}$, and

3. there exists $a_{\bullet}>0$ such that $T_{\bullet}=+\infty$ if $\left|h_{0}\right|_{\frac{1}{2}} \leq a_{\bullet}$.

4. Either the solution blows up in $\dot{H}^{\beta}$ for all $\beta>\frac{1}{2}$ at $T=T_{\bullet}$ or $T_{\bullet}=\infty$.

REMARK 2.3. If the maximal time $T_{\text {• }}$ of a solution $h$ is finite, we know that $\|h(t)\|_{\beta} \rightarrow \infty$ as $t \uparrow T_{\bullet}$ for $\beta>\frac{1}{2}$. We cannot conclude that the same is true for $\|h(t)\|_{\frac{1}{2}}$. Indeed, $h$ can be discontinuous at the maximal time $T_{\bullet}$, so either $\|h(t)\|_{\frac{1}{2}}$ is unbounded, or is bounded and discontinuous in $T_{\bullet}$.

The reason behind this is that a solution in $\dot{H}^{\frac{1}{2}}$ can be continued as long as there is a control on the quantity $K_{0}$ of the type (2.6), and this quantity is not uniformly convergent to 0 on bounded subsets of $\dot{H}^{\frac{1}{2}}$. In different words, $K_{0}$ can be controlled as long as one can control the way the mass of $h(0)$ is partitioned among Fourier modes.

The proof of this theorem is developed in several steps, which we will prove in the remainder of this section.

First, we prove existence and uniqueness (together with the global existence statement). Then we prove an analogous result in $\dot{H}^{\beta}$, for all $\beta>\frac{1}{2}$. By a standard bootstrap technique, this implies the smoothness of solutions.

Let $A$ be the operator $\partial_{x}^{4}$ with domain $\dot{H}^{4}$. It is a standard result that $A$ generates an analytic semigroup. Using for example the Fourier series expansion, it is easy to verify that

$$
\left\|A^{\gamma} \mathrm{e}^{-t A}\right\|_{\mathcal{L}\left(\dot{H}^{\beta}\right)} \leq c_{\gamma} t^{-\gamma}
$$

for every $t>0$, where $\gamma \geq 0$ and $\beta \in \mathbb{R}$. By $\mathcal{L}\left(\dot{H}^{\beta}\right)$ we denote the space of linear bounded operators on $\dot{H}^{\beta}$. Moreover, it is easy to verify that the norm $\left|A^{\frac{\beta}{4}} \cdot\right|_{L^{2}}$, which we use several times in the paper, coincides with the standard norm (2.2) on $\dot{H}^{\beta}$.

Proposition A.4 implies that for $\alpha \in\left(0, \frac{1}{2}\right)$,

$$
\left|A^{\frac{1}{8}(4 \alpha-5)}\left(h_{x}^{2}\right)_{x x}\right|_{L^{2}} \leq c_{\alpha}|h|_{1+\alpha}^{2}
$$

(just apply the proposition with $\alpha=\beta, \gamma=\frac{1}{2}-2 \alpha$ and use the dual formulation of the $L^{2}$ norm).

Consider now the right hand side of the mild formulation,

$$
\mathcal{F}(h)(t)=\mathrm{e}^{-t A} h_{0}+\int_{0}^{t} \mathrm{e}^{-(t-s) A}\left(h_{x}^{2}\right)_{x x}(s) d s,
$$


and define

$$
\begin{gathered}
K_{0}(t)=\sup _{s \in(0, t]}\left(s^{\frac{1}{8}(2 \alpha+1)}\left|\mathrm{e}^{-s A} h_{0}\right|_{1+\alpha}\right), \quad \text { for } h_{0} \in \dot{H}^{\frac{1}{2}}, \\
K(t, h)=\sup _{s \in(0, t]}\left(s^{\frac{1}{8}(2 \alpha+1)}|h(s)|_{1+\alpha}\right), \quad \text { for } h \in \mathcal{S}_{\alpha}(T), t \in[0, T] .
\end{gathered}
$$

The triangle inequality easily implies that $K(t, h+k) \leq K(t, h)+K(t, k)$. The following lemma analyses the term $K_{0}$.

LEMMA 2.4. If $h_{0} \in \dot{H}^{\frac{1}{2}}$, then

$$
K_{0}(t) \rightarrow 0 \quad \text { as } t \rightarrow 0
$$

Furthermore, for each $\beta \in\left[\frac{1}{2}, 1+\alpha\right]$ there is a constant $c_{\beta}>0$ such that

$$
K_{0}(t) \leq c_{\beta} t^{\frac{1}{8}(2 \beta-1)}\left|h_{0}\right|_{\beta}
$$

Proof. By assumption $A^{\frac{1}{8}} h_{0} \in L^{2}$, hence by Lemma C.1,

$$
s^{\frac{1}{8}(2 \alpha+1)}\left|\mathrm{e}^{-s A} h_{0}\right|_{1+\alpha}=\left|s^{\frac{1}{8}(2 \alpha+1)} A^{\frac{1}{8}(1+2 \alpha)} \mathrm{e}^{-s A} A^{\frac{1}{8}} h_{0}\right|_{L^{2}} \longrightarrow 0 .
$$

as $s \rightarrow 0$. Since by $(2.3)$,

$$
K_{0}(t)=\sup _{s \in(0, t]} s^{\frac{1}{8}(2 \alpha+1)}\left|A^{\frac{1}{4}(1+\alpha-\beta)} \mathrm{e}^{-s A} A^{\frac{\beta}{4}} h_{0}\right|_{L^{2}} \leq c_{\beta} t^{\frac{1}{8}(2 \beta-1)}\left|h_{0}\right|_{\beta},
$$

the second claim follows.

Now we proceed to find a solution to the equation $h=\mathcal{F}(h)$.

LEMMA 2.5. There is a small number $\delta>0$ depending only on $\alpha$ such that the following statement is true. For all $h_{0} \in \dot{H}^{\frac{1}{2}}$ there exists a time $T>0$ (sufficiently small) such that the map $\mathcal{F}$ is a contraction on $\mathcal{S}_{\alpha}^{\delta}(T)$.

Proof. First we show that $\mathcal{F}$ maps $\mathcal{S}_{\alpha}^{\delta}$ into itself for $T$ and $\delta$ sufficiently small. To be more precise, there is a number $c_{\alpha}>0$ such that for all $t \in[0, T]$ and all $h \in \mathcal{S}_{\alpha}^{\delta}$

$$
K(t, \mathcal{F}(h)) \leq K_{0}(t)+c_{\alpha} K(t, h)^{2} \leq K_{0}(T)+c_{\alpha} \delta^{2} .
$$

Thus for $\delta \leq\left(2 c_{\alpha}\right)^{-1}$ and $T$ sufficiently small $\mathcal{F}$ maps $\mathcal{S}_{\alpha}^{\delta}$ into itself.

In order to prove (2.8) we consider

$$
|\mathcal{F}(h)(t)|_{1+\alpha} \leq\left|\mathrm{e}^{-t A} h_{0}\right|_{1+\alpha}+\int_{0}^{t}\left|\mathrm{e}^{-(t-s) A}\left(h_{x}^{2}\right)_{x x}\right|_{1+\alpha} d s=I_{0}+I_{1} .
$$

For the first term,

$$
t^{\frac{1}{8}(2 \alpha+1)} I_{0}=t^{\frac{1}{8}(2 \alpha+1)}\left|\mathrm{e}^{-t A} h_{0}\right|_{1+\alpha} \leq K_{0}(T) \rightarrow 0
$$

as $T \rightarrow 0$, by $(2.6)$.

For the second term we use (2.4), as well as (2.3), to obtain

$$
\begin{aligned}
I_{1} & =\int_{0}^{t}\left|A^{\frac{5-4 \alpha}{8}+\frac{1+\alpha}{4}} \mathrm{e}^{-(t-s) A} A^{\frac{1}{8}(4 \alpha-5)}\left(h_{x}^{2}\right)_{x x}\right|_{L^{2}} d s \\
& \leq c K(t, h)^{2} \int_{0}^{t} s^{-\frac{1}{4}(2 \alpha+1)}(t-s)^{-\frac{1}{8}(7-2 \alpha)} d s \\
& =C_{\alpha} t^{-\frac{1}{8}(1+2 \alpha)} K(t, h)^{2},
\end{aligned}
$$


where $C_{\alpha}=c B\left(\frac{1}{4}(3-2 \alpha), \frac{1}{8}(1+2 \alpha)\right)$ and $B(x, y)=\int_{0}^{1} t^{x-1}(1-t)^{y-1} d t$ is the Beta function.

Now let us show that $\mathcal{F}$ is a contraction on $\mathcal{S}_{\alpha}$. If $h, k \in \mathcal{S}_{\alpha}$, then by following essentially the above estimate of $I_{1}$, one can derive the following estimate

$$
K(T, \mathcal{F}(h)-\mathcal{F}(k)) \leq C_{\alpha} K(T, h-k) K(T, h+k) \leq 2 \delta C_{\alpha} K(T, h-k)
$$

Thus $\mathcal{F}$ is a contraction if $\delta \leq\left(4 C_{\alpha}\right)^{-1}$.

The following corollary is obvious, if we use (2.7) for $\beta>\frac{1}{2}$. The same conclusion cannot be drawn in the case $\beta=\frac{1}{2}$ ( see Remark 2.3).

Corollary 2.6. If $h_{0} \in \dot{H}^{\beta}$ for $\beta>\frac{1}{2}$, then the time $T$ in the previous lemma depends only on the quantity $\left|h_{0}\right|_{\beta}$.

Thus, as long as a solution is bounded in any $\dot{H}^{\beta}$ with $\beta>\frac{1}{2}$, the interval of existence can be extended by a fixed length $T$, which depends only on the bounding constant.

The next lemma shows that the solution to the fixed point $h=\mathcal{F}(h)$ in $\mathcal{S}_{\alpha}$ is continuous with values in $H^{1 / 2}$.

LEMmA 2.7. If $h \in \mathcal{S}_{\alpha}(T)$, then $\mathcal{F}(h) \in C^{0}\left((0, T], \dot{H}^{\frac{1}{2}}\right)$.

Proof. Obviously, it is enough to show that $\mathcal{F}(h)$ is continuous in $t=0$. First, $\mathrm{e}^{-t A} h_{0} \rightarrow h_{0}$ in $\dot{H}^{\frac{1}{2}}$ by continuity of the semigroup. It remains to show that

$$
\int_{0}^{t} \mathrm{e}^{-(t-s) A}\left(h_{x}(s)^{2}\right)_{x x} d s \longrightarrow 0
$$

in $\dot{H}^{\frac{1}{2}}$ for $t \rightarrow 0$. We know already by $(2.4)$ that $f(s)=s^{\frac{1}{4}(2 \alpha+1)} A^{\frac{1}{8}(4 \alpha-5)}\left(h_{x}^{2}\right)_{x x}$ is bounded in $L^{2}$ for $s \in(0, T]$ with $|f(s)|_{L^{2}} \leq c K(s, h)^{2}$. Thus from Lemma C.1,

$$
\int_{0}^{t} s^{-\frac{1}{4}(2 \alpha+1)} A^{-\frac{1}{8}(4 \alpha-5)+\frac{1}{8}} \mathrm{e}^{-(t-s) A} f(s) d s \longrightarrow 0
$$

in $L^{2}$, as $t \rightarrow 0$.

Proposition 2.8. Given $h_{0} \in \dot{H}^{\frac{1}{2}}$ and $\alpha \in\left(0, \frac{1}{2}\right)$, there exist $T_{0}>0$ and $\delta_{0}$, depending only on $\alpha$ and $h_{0}$, such that there is a unique solution in $\mathcal{S}_{\alpha}^{\delta_{0}}\left(T_{0}\right)$ to problem (1.1) starting at $h_{0}$.

Moreover, the solution is in $C^{0}\left(\left[0, T_{0}\right), \dot{H}^{1 / 2}\right)$ and there exists $a_{0}>0$ small enough such that, if $\left|h_{0}\right|_{1 / 2} \leq a_{0}$, then $T_{0}=\infty$.

Proof. Most of the proof is already done. We only need to prove the last statement of the proposition. By $(2.7), K_{0}(t) \leq c_{0}\left|h_{0}\right|_{\frac{1}{2}}$, so that, if we choose $a_{0} \leq\left(c_{0} c_{\alpha}\right)^{-1}$ (where $c_{\alpha}$ is the constant in formula (2.8)) and $K=\left(2 c_{\alpha}\right)^{-1}(1-$ $\left.\sqrt{1-c_{0} c_{\alpha} a_{0}}\right)$, by $(2.8)$ it follows that, for $K(t, h) \leq K$,

$$
K(t, \mathcal{F}(h)) \leq K_{0}(t)+c_{\alpha} K(t, h)^{2} \leq c_{0} a_{0}+c_{\alpha} K^{2} \leq K,
$$

independently of $t$. Hence, $T_{0}=\infty$.

REMARK 2.9 (Criticality of $\mathcal{S}_{\alpha}(T)$ ). Following the same notation used in Section 2, we have that if $h \in \mathcal{S}_{\alpha}(T)$, then $h_{\lambda} \in \mathcal{S}_{\alpha}\left(T_{\lambda}\right)$ and $K\left(T_{\lambda}, h_{\lambda}\right)$ scales as $\lambda^{\frac{1}{8}(1-6 \alpha)} K(T, h)$. So, apparently, the $\|\cdot\|_{\alpha, T}$ does not obey the scaling heuristic. On the other hand, this information is of no use. Indeed, the scaling behaviour is hidden, as it is shown by Lemma 2.13 below, where the boundedness in a space 
which is almost $\mathcal{S}_{\alpha}$ implies boundedness in the critical space $L^{q}\left(0, T ; \dot{H}^{1+\alpha}\right)$, with $q=\frac{8}{1+2 \alpha}$.

Next, the case of more regular initial conditions is considered. The result is stated for integer exponents only, since for showing regularity the present version is sufficient (we already know that solutions with initial value in $\dot{H}^{\frac{1}{2}}$ are continuous in $\dot{H}^{1}$ ). It is easy to adapt the proposition to non-integer exponents, with some slight changes.

Proposition 2.10. Let $n \in \mathbf{N}, n \geq 1$. Given an arbitrary $h_{0} \in \dot{H}^{n}$, there exist $T>0$ and a solution $h \in C\left([0, T) ; \dot{H}^{n}\right) \cap L_{l o c}^{2}\left([0, T) ; \dot{H}^{n+2}\right)$ to problem (1.1), with initial condition $h_{0}$.

Proof. We only prove the core a-priori estimate for the Theorem. Existence of a solution can be proven by means of Proposition 2.8 or by an approximation procedure (such as finite-dimensional approximations).

Start by $n=1$,

$$
\frac{d}{d t}|h|_{1}^{2}=2\left\langle h, \partial_{t} h\right\rangle_{1}=-2|h|_{3}^{2}-2\left\langle h_{x x},\left(h_{x}^{2}\right)_{x x}\right\rangle .
$$

By integration by parts and Sobolev, interpolation and Young's inequalities, we get

$$
\begin{aligned}
2\left\langle h_{x x},\left(h_{x}^{2}\right)_{x x}\right\rangle & =-2\left\langle h_{x x x}, 2 h_{x} h_{x x}\right\rangle \\
\text { (by Hölder's inequality) } & \leq 2\left|h_{x x x}\right|_{L^{2}}\left|h_{x}\right|_{L^{6}}\left|h_{x x}\right|_{L^{3}} \\
\text { (by Sobolev embedding) } & \leq c|h|_{3}|h|_{\frac{4}{3}}|h|_{\frac{13}{6}} \\
\text { (by interpolation) } & \leq c|h|_{3}^{\frac{7}{4}}|h|_{1}^{\frac{5}{4}} \\
\text { (by Young's inequality) } & \leq|h|_{3}^{2}+c|h|_{1}^{10} .
\end{aligned}
$$

In conclusion, if we define $\varphi(t)=|h(t)|_{1}^{2}+\int_{0}^{t}|h|_{3}^{2} d s$, the above inequality reads

$$
\dot{\varphi}=\frac{d}{d t}|h|_{1}^{2}+|h|_{3}^{2} \leq c|h|_{1}^{10} \leq c \varphi^{5}
$$

and by solving the differential inequality, we have a time $T$ such that $h$ is bounded in $C\left([0, T) ; \dot{H}^{1}\right)$ and in $L_{\mathrm{loc}}^{2}\left([0, T) ; \dot{H}^{3}\right)$.

The method is similar for $n \geq 2$. By computing the derivative of $|h(t)|_{n}^{2}$, it turns out that it is necessary to estimate the term originating from the nonlinear part. By integration by parts and Leibniz formula,

$$
\begin{aligned}
2\left\langle D^{2 n} h,\left(h_{x}^{2}\right)_{x x}\right\rangle & =2\left\langle D^{n+2} h, D^{n}\left(h_{x}^{2}\right)\right\rangle \\
& =2 \sum_{k=0}^{n}\left(\begin{array}{l}
n \\
k
\end{array}\right)\left\langle D^{n+2} h,\left(D^{k+1} h\right)\left(D^{n+1-k} h\right)\right\rangle .
\end{aligned}
$$

By applying Hölder's inequality and Sobolev embedding, the above sum can be estimated as above. All terms $|h|_{a}$ with $a \leq n$ can be controlled by $|h|_{n}$, while all terms with $a \in(n, n+2)$ can be controlled by $|h|_{n}$ and $|h|_{n+2}$ by interpolation. We finally get the estimate

$$
\frac{d}{d t}|h|_{n}^{2}+2|h|_{n+2}^{2} \leq|h|_{n+2}^{2}+c_{n}|h|_{n}^{a_{n}}
$$

with suitable $c_{n}$ and $a_{n}$, depending only on $n$. By solving, as above, the implied differential inequality, the solution $h$ turns out to be bounded in $C\left([0, T) ; \dot{H}^{n}\right)$ and in $L_{\mathrm{loc}}^{2}\left([0, T) ; \dot{H}^{n+2}\right)$. 
Everything is now ready to carry on the proof of the main theorem of this section.

Proof of Theorem 2.2. The existence of solutions with initial condition in $\dot{H}^{1 / 2}$, as well as the $T_{\bullet}=\infty$ statement, follow from Proposition 2.8.

The regularity statement (1) follows from Proposition 2.10. Indeed, by Proposition 2.8, a solution starting in $\dot{H}^{1 / 2}$ is continuous with values in $\dot{H}^{1}$. By applying Proposition 2.10 on each $h(t) \in \dot{H}^{1}$, for $t \leq T_{\bullet}$, it follows that the solution is in $C\left(\left(0, T_{\bullet}\right) ; \dot{H}^{1}\right)$ and $L_{\text {loc }}^{2}\left(\left(0, T_{\bullet}\right) ; \dot{H}^{3}\right)$. The last statement implies that $h(t) \in \dot{H}^{3}$, for almost every $t \in\left(0, T_{\bullet}\right)$ and so Proposition 2.10 can be used with $n=3$, and so on. By iterating the procedure, it follows that $h \in C\left(\left(0, T_{\bullet}\right) ; \dot{H}^{\beta}\right)$ for all $\beta \geq 1$. Time regularity now follows from this space regularity and the mild form (2.5).

The energy equality in $(\mathbf{2})$ is now easy using the space-time regularity in $\left(0, T_{\boldsymbol{\bullet}}\right)$ and the continuity at $t=0$ in the $L^{2}$ norm.

2.2. Uniqueness among weak solutions. A weak solution to equation (1.1) is a function $h \in L_{\mathrm{loc}}^{\infty}\left([0, \infty) ; L^{2}\right) \cap L_{\mathrm{loc}}^{2}\left([0, \infty) ; \dot{H}^{2}\right)$ that satisfies the equation in distributions. Existence of such solutions for all initial data in $L^{2}$ has been established in $[25]$ (or $[5,7]$ ). The following theorem shows that the solutions provided by Theorem 2.2 are unique in the class of all weak solutions $h$ that satisfy the energy inequality (1.3).

TheOREM 2.11. Let $h_{0} \in \dot{H}^{\frac{1}{2}}$ and let $h \in C\left(\left[0, T_{\bullet}\right) ; \dot{H}^{\frac{1}{2}}\right)$ be the solution to (1.1) provided by Theorem 2.2 and defined up to its maximal time $T_{\bullet}$. Then every weak solution to (1.1) starting at $h(0)$ coincides with $h$ on $\left[0, T_{\bullet}\right)$.

In order to prove the theorem, we shall proceed in several steps. We will essentially prove that any solution in $\mathcal{S}_{\alpha}(T)$ with an additional integrability condition is unique in the class of weak solutions (Proposition 2.12 below). Then we prove that solutions in $\mathcal{S}_{\alpha}(T)$ satisfy the additional condition (Lemma 2.13 and 2.14). It is worth remarking that the additional integrability condition (2.9) turns out to correspond to the critical space $L^{\frac{8}{1+2 \alpha}}\left(0, T ; H^{1+\alpha}\right)$ (see Section 3.1).

Proposition 2.12. Let $h \in \mathcal{S}_{\alpha}(T)$ be a solution to (1.1) and assume moreover that

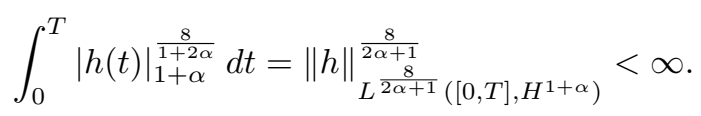

Then $h$ is the unique weak solution starting at $h(0)$.

Proof. Let $k$ be any weak solution starting at $h(0)$. Since $h \in C^{\infty}((0, T] \times$ $[0, L])$ and $h$ is continuous in $\dot{H}^{\frac{1}{2}}$, it follows that

$$
\langle h(t), k(t)\rangle+2 \int_{0}^{t}\left\langle h_{x x}, k_{x x}\right\rangle d s=|h(0)|_{L^{2}}^{2}-\int_{0}^{t} \int_{0}^{L}\left(h_{x x} k_{x}^{2}+k_{x x} h_{x}^{2}\right) d x d s
$$

which, together with the energy inequality for $k$ and the energy equality (see Theorem 2.2) for $h$ implies that the difference $w=h-k$ satisfies the following energy 
inequality,

$$
\begin{aligned}
|w(t)|_{L^{2}}^{2}+2 \int_{0}^{t}\left|w_{x x}\right|_{L^{2}}^{2} d s & \leq 2 \int_{0}^{t} \int_{0}^{L}\left(h_{x x} k_{x}^{2}+k_{x x} h_{x}^{2}\right) d x d s \\
& =4 \int_{0}^{t} \int_{0}^{L} k_{x} w_{x} w_{x x} d x d t
\end{aligned}
$$

where we have used (1.2) since

$$
h_{x x} k_{x}^{2}+k_{x x} h_{x}^{2}=2 k_{x} w_{x} w_{x x}+w_{x x} w_{x}^{2}+h_{x x} h_{x}^{2}+k_{x x} k_{x}^{2} .
$$

The conclusion now follows from the assumption (2.9) and Gronwall's lemma, since

$$
\begin{aligned}
\int_{0}^{L} k_{x} w_{x} w_{x x} d x & \leq\left|w_{x x}\right|_{L^{2}}\left|w_{x}\right|_{L^{\frac{1}{\alpha}}}\left|k_{x}\right|_{L^{\frac{2}{1-2 \alpha}}} \\
& \leq c|w|_{2}\left|w_{x}\right|_{\frac{1-2 \alpha}{2}}\left|k_{x}\right|_{\alpha} \\
& \leq c|w|_{2}|k|_{1+\alpha}|w|_{\frac{3-2 \alpha}{2}} \\
& \leq|w|_{2}^{2}+c|k|_{1+\alpha}^{\frac{8}{1+2 \alpha}}|w|_{L^{2}}^{2}
\end{aligned}
$$

where we have used Hölder's inequality (with exponents $2, \frac{1}{\alpha}$, and $\frac{2}{1-2 \alpha}$ ), the Sobolev embeddings $H^{\alpha} \subset L^{\frac{2}{1-2 \alpha}}$ and $H^{\frac{1-2 \alpha}{2}} \subset L^{\frac{1}{\alpha}}$, interpolation of $H^{\frac{3-2 \alpha}{2}}$ between $L^{2}$ and $H^{2}$, and finally Young's inequality.

Since there seems to be no obvious way to prove property (2.9) for any arbitrary element of $\mathcal{S}_{\alpha}(T)$, we are led to prove this additional regularity for solutions of (2.5). To this end, define for $T>0$ and $\alpha \in\left(0, \frac{1}{2}\right)$,

$$
\left(\|u\|_{\alpha, T}^{\star}\right)^{2}=\sum_{k \neq 0} k^{2(1+\alpha)}\left(\sup _{s \leq T}\left\{s^{\frac{1}{8}(1+2 \alpha)}\left|u_{k}(s)\right|\right\}\right)^{2}
$$

and

$$
\mathcal{S}_{\alpha}^{\star}(T)=\left\{u \in \mathcal{S}_{\alpha}(T):\|u\|_{\alpha, T}^{\star}<\infty\right\} .
$$

Assuming that $\mathcal{S}_{\alpha}^{\star}(T) \subset \mathcal{S}_{\alpha}(T)$ is not restrictive, since it is easy to verify that $\|\cdot\|_{\alpha, T} \leq\|\cdot\|_{\alpha, T}^{\star}$.

Lemma 2.13. If $h \in \mathcal{S}_{\alpha}^{\star}(T)$, then $\mathcal{F}(h)$ satisfies $(2.9)$ on $[0, T]$.

Proof. We write $\mathcal{F}(h)(t)=H_{0}(t)+H_{1}(t)$ where $H_{0}(t)=\mathrm{e}^{-t A} h(0)$ and $H_{1}$ contains the nonlinearity. Now,

$$
\left|H_{0}(t)\right|_{1+\alpha}^{2}=\sum_{k \neq 0} k^{2(1+\alpha)} \mathrm{e}^{-2 c t k^{4}}\left|h_{k}(0)\right|^{2},
$$

and so, if $\varphi \in L^{q}(0, T)$ with $p=\frac{4}{1+2 \alpha}$ and $\frac{1}{p}+\frac{1}{q}=1$,

$$
\begin{aligned}
\int_{0}^{T} \varphi(t)\left|H_{0}(t)\right|_{1+\alpha}^{2} d t & =\sum_{k \neq 0} k^{2(1+\alpha)}\left|h_{k}(0)\right|^{2} \int_{0}^{T} \varphi(t) \mathrm{e}^{-2 c t k^{4}} d t \\
& \leq\|\varphi\|_{L^{q}} \sum_{k \neq 0} k^{2(1+\alpha)}\left|h_{k}(0)\right|^{2}\left(\int_{0}^{T} \mathrm{e}^{-2 c t p k^{4}} d t\right)^{\frac{1}{p}} \\
& \leq c_{p}\|\varphi\|_{L^{q}}|h(0)|_{\frac{1}{2}}^{2} .
\end{aligned}
$$


By duality, the $L^{\frac{8}{1+2 \alpha}}$ norm of $\left|H_{0}\right|_{1+\alpha}$ is finite. The second term is more delicate, we shall proceed as in the proof of Proposition A.4,

$$
\begin{aligned}
\left|H_{1}(t)\right|_{1+\alpha}^{2} & =\sum_{k \neq 0} k^{2(1+\alpha)}\left(\int_{0}^{t} \mathrm{e}^{-c(t-s) k^{4}}\left[\left(h_{x}^{2}\right)_{x x}\right]_{k}\right)^{2} \\
& =\sum_{k \neq 0} k^{2(3+\alpha)}\left(\sum_{l+m=k}|l m| \int_{0}^{t} \mathrm{e}^{-c(t-s) k^{4}}\left|h_{l}(s) h_{m}(s)\right| d s\right)^{2} \\
& \leq \sum_{k \neq 0} k^{2(3+\alpha)}\left(\sum_{l+m=k}|l m| h_{l}^{\star} h_{m}^{\star}\right)^{2}\left(\int_{0}^{t} \mathrm{e}^{-c(t-s) k^{4}} s^{-\frac{1+2 \alpha}{4}} d s\right)^{2},
\end{aligned}
$$

where $h_{k}^{\star}=\sup _{s \leq T} s^{\frac{1+2 \alpha}{8}}\left|h_{k}(s)\right|$. Hence, for every $\varphi \in L^{q}(0, T)$,

$$
\begin{aligned}
& \int_{0}^{T} \varphi(t)\left|H_{1}(t)\right|_{1+\alpha} d t \leq \\
& \quad \leq \sum_{k \neq 0} k^{2(3+\alpha)}\left(\sum_{l+m=k}|l m| h_{l}^{\star} h_{m}^{\star}\right)^{2} \int_{0}^{T} \varphi(t)\left(\int_{0}^{t} \mathrm{e}^{-c(t-s) k^{4}} s^{-\frac{1+2 \alpha}{4}}\right)^{2} d t .
\end{aligned}
$$

Suppose that

$$
\int_{0}^{T} \varphi(t)\left(\int_{0}^{t} \mathrm{e}^{-c(t-s) k^{4}} s^{-\frac{1+2 \alpha}{4}} d s\right)^{2} d t \leq c\|\varphi\|_{L^{q}} k^{2 \alpha-7} .
$$

In this case we can proceed as in the proof of Proposition A.4 (where the $h_{k}^{\star}$ replace the Fourier components and $\gamma=\frac{1}{2}-2 \alpha$ ) to obtain that

$$
\int_{0}^{T} \varphi(t)\left|H_{1}(t)\right|_{1+\alpha} d t \leq c\|\varphi\|_{L^{q}}\left(\|h\|_{\alpha, T}^{\star}\right)^{2}
$$

and, again by duality, boundedness of $\mathcal{F}(h)$.

So, everything boils down to proving (2.10). Using Hölder's inequality and (twice) a change of variables,

$$
\begin{aligned}
\int_{0}^{T} \varphi(t) & \left(\int_{0}^{t} \mathrm{e}^{-c(t-s) k^{4}} s^{-\frac{1+2 \alpha}{4}} d s\right)^{2} d t \leq \\
& \leq\|\varphi\|_{L^{q}}\left[\int_{0}^{T}\left(\int_{0}^{t} \mathrm{e}^{-c(t-s) k^{4}} s^{-\frac{1+2 \alpha}{4}} d s\right)^{2 p} d t\right]^{\frac{1}{p}} \\
& \leq\|\varphi\|_{L^{q}} k^{2 \alpha-7}\left[\int_{0}^{k^{4} T}\left(\int_{0}^{t} \mathrm{e}^{-c(t-s)} s^{-\frac{1+2 \alpha}{4}} d s\right)^{2 p} d t\right]^{\frac{1}{p}} .
\end{aligned}
$$

It is elementary now to verify that the integral on the right-hand side is uniformly bounded in $k$ and $T$ (by bounding the integral on $\left[0, k^{4} T\right]$ with the same integral on $[0, \infty))$. Indeed,

$$
\int_{0}^{\frac{t}{2}} \mathrm{e}^{-c(t-s)} s^{-\frac{1+2 \alpha}{4}} d s \leq c t^{\frac{3-2 \alpha}{4}} \mathrm{e}^{-\frac{c}{2} t},
$$

whose $p$ th-power is integrable at $\infty$, as well as

$$
\int_{\frac{t}{2}}^{t} \mathrm{e}^{-c(t-s)} s^{-\frac{1+2 \alpha}{4}} d s \leq c t^{-\frac{2 \alpha+1}{4}}\left(1-\mathrm{e}^{-\frac{c}{2} t}\right) \leq c t^{-\frac{2 \alpha+1}{4}},
$$

since $t^{-2 p \frac{2 \alpha+1}{4}}=t^{-2}$. 
The final step is to prove that solutions exist in the smaller space $\mathcal{S}_{\alpha}^{\star}$. This coincides then with the unique weak solution and the solution given by Theorem 2.2.

Lemma 2.14. Let $h_{0} \in \dot{H}^{\frac{1}{2}}$ and $\alpha \in\left(0, \frac{1}{2}\right)$. Then there is $T_{\star}>0$ such that there exists a unique solution $h$ in $\mathcal{S}_{\alpha}^{\star}\left(T_{\star}\right)$.

ProOF. The proof is essentially a fixed point argument, as in Proposition 2.8. Let

$$
H_{0}(t)=\mathrm{e}^{-t A} h(0) \quad \text { and } \quad H_{1}=\mathcal{F}(h)-H_{0},
$$

then it is sufficient to show the following facts:

1. $\left\|H_{0}\right\|_{\alpha, T}^{\star} \leq\|h(0)\|_{\frac{1}{2}}$,

2. $\left\|H_{0}\right\|_{\alpha, T}^{\star} \longrightarrow 0$ as $T \rightarrow 0$,

3. there is $c>0$ (independent of $T$ ) such that for all $h \in \mathcal{S}_{\alpha}^{\star}(T),\|\mathcal{F}(h)\|_{\alpha, T}^{\star} \leq$ $\left\|H_{0}\right\|_{\alpha, T}^{\star}+c\left(\|h\|_{\alpha, T}^{\star}\right)^{2}$, and

4. there is $c>0$ (independent of $T$ ) such that for all $g, h \in \mathcal{S}_{\alpha}^{\star}(T)$,

$$
\|\mathcal{F}(g)-\mathcal{F}(h)\|_{\alpha, T}^{\star} \leq c\|g-h\|_{\alpha, T}^{\star}\|g+h\|_{\alpha, T}^{\star} .
$$

Notice that

$$
\sup _{s \leq T} s^{\frac{1+2 \alpha}{8}}\left|\left[H_{0}(t)\right]_{k}\right|=\left|h_{k}(0)\right| \sup _{s \leq T} s^{\frac{1+2 \alpha}{8}} \mathrm{e}^{-c s k^{4}} \leq c k^{-\frac{1+2 \alpha}{2}}\left|h_{k}(0)\right|
$$

and so

$$
\left(\left\|H_{0}\right\|_{\alpha, T}^{\star}\right)^{2} \leq \sum_{k \neq 0} k^{2(1+\alpha)}\left|h_{k}(0)\right|^{2} c k^{-(1+2 \alpha)} \leq c\|h(0)\|_{\frac{1}{2}}^{2} .
$$

In order to prove the second property, we have to refine the previous calculation. Fix $\varepsilon>0$ such that $\varepsilon \leq c_{\alpha}$ (where $c_{\alpha}^{4}$ is the point where the function $s^{\frac{1+2 \alpha}{8}} \mathrm{e}^{-s}$ attains its maximum), then

$$
\begin{aligned}
\left(\left\|H_{0}\right\|_{\alpha, T}^{\star}\right)^{2} & =\left(\sum_{|k| \leq \varepsilon T^{-\frac{1}{4}}}+\sum_{|k|>\varepsilon T^{-\frac{1}{4}}}\right) k^{2(1+\alpha)}\left|h_{k}(0)\right|^{2} \sup _{s \in[0, T]}\left\{s^{\frac{1+2 \alpha}{4}} \mathrm{e}^{-2 c s k^{4}}\right\} \\
& \leq \sum_{|k| \leq \varepsilon T^{-\frac{1}{4}}}\left(k^{4} T\right)^{\frac{1+2 \alpha}{4}}\left|k \| h_{k}(0)\right|^{2}+\sum_{|k|>\varepsilon T^{-\frac{1}{4}}} k\left|h_{k}(0)\right|^{2} \\
& \leq \varepsilon^{1+2 \alpha}\|h(0)\|_{\frac{1}{2}}^{2}+c \sum_{|k|>\varepsilon T^{-\frac{1}{4}}}|k|\left|h_{k}(0)\right|^{2} .
\end{aligned}
$$

Now, $\lim \sup _{T \rightarrow 0}\left\|H_{0}\right\|_{\alpha, T}^{\star} \leq \varepsilon^{1+2 \alpha}\|h(0)\|_{\frac{1}{2}}^{2}$ and, as $\varepsilon \downarrow 0$, the conclusion follows.

In order to prove the last fact, we follow the proof of Lemma 2.13,

$$
\left|\left(H_{1}\right)_{k}(t)\right| \leq k^{2} \sum_{l+m=k}|l m| h_{l}^{\star} h_{m}^{\star} \cdot \int_{0}^{t} \mathrm{e}^{-c(t-s) k^{4}} s^{-\frac{1+2 \alpha}{4}} d s
$$

and so

$$
\sup _{t \in[0, T]}\left\{t^{\frac{1+2 \alpha}{8}}\left|\left(H_{1}\right)_{k}(t)\right|\right\} \leq k^{2} \sum_{l+m=k}|\operatorname{lm}| h_{l}^{\star} h_{m}^{\star} \sup _{t \in[0, T]}\left\{t^{\frac{1+2 \alpha}{8}} \int_{0}^{t} \mathrm{e}^{-c(t-s) k^{4}} s^{-\frac{1+2 \alpha}{4}} d s\right\} .
$$

Assume that the supremum in the above formula is bounded by $c_{\alpha} k^{-\frac{7-2 \alpha}{2}}$ (we shall prove this later), then, as in the proof of Proposition A.4,

$$
\left(\left\|H_{1}\right\|_{\alpha, T}^{\star}\right)^{2} \leq c_{\alpha} \sum_{k \neq 0} k^{6+2 \alpha}\left(k^{-\frac{7-2 \alpha}{2}} \sum_{l+m=k}|l m| h_{l}^{\star} h_{m}^{\star}\right)^{2} \leq c\left(\|h\|_{\alpha, T}^{\star}\right)^{4} .
$$


As it regards the remaining bound, we use $\mathrm{e}^{-c(t-s) k^{4}} \leq c_{\alpha}\left[k^{4}(t-s)\right]^{-\frac{7-2 \alpha}{8}}$, in order to get

$$
\sup _{t \in[0, T]}\left\{t^{\frac{1+2 \alpha}{8}} \int_{0}^{t} \mathrm{e}^{-c(t-s) k^{4}} s^{-\frac{1+2 \alpha}{4}} d s\right\} \leq c_{\alpha} B\left(\frac{3-2 \alpha}{4}, \frac{1+2 \alpha}{8}\right) k^{-\frac{7-2 \alpha}{2}},
$$

and $B$ is the Beta function.

The proof of the last fact is similar. Indeed, if $g, h \in \mathcal{S}_{\alpha}^{\star}(T)$, then

$$
\begin{aligned}
\left|[\mathcal{F}(g)(t)-\mathcal{F}(h)(t)]_{k}\right| & \leq k^{2} \int_{0}^{t} \mathrm{e}^{-c(t-s) k^{4}}\left|\left[(g-h)_{x}(g+h)_{x}\right]_{k}\right| d s \\
& \leq k^{2} \sum_{l+m=k}|l m| \int_{0}^{t} \mathrm{e}^{-c(t-s) k^{4}}\left|\left(g_{l}-h_{l}\right)\left(g_{m}+h_{m}\right)\right| d s
\end{aligned}
$$

and so, by proceeding as above, the last fact follows.

Proof of Theorem 2.11. Given $h(0) \in \dot{H}^{\frac{1}{2}}$, let $h \in C\left(\left[0, T_{\bullet}\right) ; \dot{H}^{\frac{1}{2}}\right)$ be the solution provided by Theorem 2.2 and fix $T<T_{\text {. }}$. By Lemma 2.14 we know that $h \in \mathcal{S}_{\alpha}^{\star}\left(T_{\star}\right)$, so Lemma 2.13 implies that $h$ satisfies the integrability condition (2.9) on $\left[0, T_{\star}\right]$. By property $(\mathbf{1})$ of Theorem $2.2, h$ satisfies trivially $(2.9)$ on $\left[T_{\star}, T\right]$. So Proposition 2.12 applies and the conclusion follows.

\section{Regularity}

3.1. Criticality. In this section, we carry out the program described in the beginning of the previous section. We will find spaces $Y_{T}$ such that boundedness in these spaces imply uniqueness for solutions starting in $H^{1 / 2}$.

Let us first discuss regularity criteria in Lebesgue spaces. Set $T_{\lambda}=\lambda^{-4} T$ and $L_{\lambda}=\lambda^{-1} L$ and consider the space $X(\lambda)=L^{q}\left(0, T_{\lambda} ; L^{p}\left(0, L_{\lambda}\right)\right)$, for some values of $p$ and $q$. Under the scaling (2.1) we have that

$$
\left\|h_{\lambda}\right\|_{X(\lambda)}=\lambda^{-\frac{4}{q}-\frac{1}{p}}\|h\|_{X(1)} .
$$

so that the space $L^{\infty}((0, T) \times(0, L))$ turns out to be the only critical space in this class. All other Lebesgue spaces are super-critical.

The conjecture now is that solutions in $L^{\infty}\left(0, T ; L^{\infty}(0, L)\right)$ or $C((0, T) \times(0, L))$ are unique and regular. We believe that with similar methods, as in the existence for initial conditions in $H^{1 / 2}$, one should be able to prove existence of unique local solutions. But this is much more involved, especially results like Lemma C.1 are hard to obtain in $C^{0}$ instead of $L^{2}$.

In order to consider Sobolev spaces, we set $X(\lambda)=L^{q}\left(0, T_{\lambda} ; \dot{W}^{k, p}\left(0, L_{\lambda}\right)\right)$ and

$$
\left\|h_{\lambda}\right\|_{X(\lambda)}=\lambda^{k-\frac{4}{q}-\frac{1}{p}}\|h\|_{X(1)} .
$$

(this is easy for integer $k$ and tricky for non-integer values, but it can be done). Hence, the space is critical for

$$
\frac{4}{q}+\frac{1}{p}=k
$$

In the following subsection, we will give the corresponding criteria for $p=2, k$ arbitrary and $p=4, k=1$. The extension to $k=1$ and $p$ arbitrary is straightforward and not presented here.

Let us finally remark, that in the following, we also give regularity criteria for $L^{4}\left(0, T, C^{1}(0, L)\right)$, which is also a critical space. 
3.2. Regularity Criteria. In principle the following Meta-theorem should hold: If a solution is bounded in a critical space, then it is unique, and does not have a blow up. This means that the unique local solution exists as long as at least one (hence all, as the solution is then proved to be regular) of the critical norms is finite over the time horizon. The main obstruction to the application of the meta-theorem is the boundedness of critical quantities. While there are cases (the Navier-Stokes equations in dimension two, for instance) where the a-priori estimates provide such bounds, this does not happen, as far as we know, in the problem studied here.

For simplicity, in the rest of the section we focus only on some examples and we consider solutions with sufficiently smooth initial condition, in order to have energy type estimates for the $H^{1}$-norm without any trouble at $t=0$.

We just remark that energy estimates in any other $H^{s}$-space with $s>\frac{1}{2}$ yield exactly the same result.

Theorem 3.1. Let $h_{0} \in \dot{H}^{1}$, let $h=h\left(\cdot, h_{0}\right)$ be the unique local solution started at $h_{0}$ and let $\tau\left(h_{0}\right)$ be the maximal time of $h$. Then $h$ is $C^{\infty}$ in space and time on $\left(0, \tau\left(h_{0}\right)\right)$ and for every $\alpha \in\left(\frac{1}{2}, \frac{9}{2}\right)$,

$$
\int_{0}^{\tau\left(h_{0}\right)}\|h(s)\|_{H^{\alpha}}^{\frac{8}{2 \alpha-1}} d s=\infty .
$$

Moreover,

$$
\int_{0}^{\tau\left(h_{0}\right)}\|h(s)\|_{W^{1,4}}^{16 / 3} d s=\infty \quad \text { and } \quad \int_{0}^{\tau\left(h_{0}\right)}\|h(s)\|_{C^{1}}^{4} d s=\infty .
$$

Proof. We already know by Theorem 2.2 that there is a unique local solution in $C\left((0, \tau) ; \dot{H}^{1}\right)$ for initial conditions in $\dot{H}^{1}$, which is actually smooth. Indeed $h \in C^{\infty}((0, \tau) \times(0, L))$. Furthermore, the $H^{1}$-norm blows up as $t \rightarrow \tau$.

Now fix $\alpha \in\left(\frac{1}{2}, \frac{9}{2}\right)$, then by integration by parts and the Sobolev embedding $H^{\frac{1}{6}} \subset L^{3}$,

$$
\begin{aligned}
\frac{d}{d t}|h|_{1}^{2}+2|h|_{3}^{2} & =-2\left\langle h,\left(h_{x}^{2}\right)_{x x}\right\rangle_{1}=-2\left\langle h_{x},\left(h_{x}^{2}\right)_{x x x}\right\rangle_{L^{2}} \\
& =-4 \int_{0}^{L} h_{x} h_{x x} h_{x x x} d x=2 \int_{0}^{L} h_{x x}^{3} d x \\
& \leq c|h|_{\frac{13}{6}}^{3} .
\end{aligned}
$$

By interpolation, it is easy to see that

$$
|h|_{\frac{13}{6}}^{3} \leq|h|_{1}^{\frac{2 \alpha-1}{4}}|h|_{\alpha}|h|_{3}^{\frac{9-2 \alpha}{4}},
$$

and so using Young's inequality,

$$
\frac{d}{d t}|h|_{1}^{2}+2|h|_{3}^{2} \leq|h|_{3}^{2}+c|h|_{\alpha}^{\frac{8}{2 \alpha-1}}|h|_{1}^{2}
$$

Finally, by Gronwall's lemma, the proof of the first statement is complete.

Let us turn again to (3.1). The Sobolev embedding $H^{\frac{1}{4}} \subset L^{4}$ yields

$$
\frac{d}{d t}|h|_{1}^{2}+2|h|_{3}^{2} \leq C|h|_{W^{1,4}}|h|_{2+\frac{1}{4}}|h|_{3} .
$$


Again by interpolation and Young's inequality

$$
\frac{d}{d t}|h|_{1}^{2}+2|h|_{3}^{2} \leq|h|_{3}^{2}+C|h|_{W^{1,4}}^{16 / 3}|h|_{1}^{2},
$$

which yields the result using again Gronwall's lemma.

The last claim follows similarly, using

$$
\frac{d}{d t}|h|_{1}^{2}+2|h|_{3}^{2} \leq C|h|_{C^{1}}|h|_{2}|h|_{3} .
$$

3.3. $H^{3}$-regularity. In this section we show that $h \in L^{p}\left(0, T, H^{3}\right)$ for some small $p>0$ (possibly less than 1 ). The idea is that we gain spatial regularity by paying time regularity.

TheOREM 3.2. Assume that a solution $h$ satisfies $h \in L^{r}\left(0, T, H^{1}\right)$ for some $r \in(0,8)$. Then $h \in L^{r / 5}\left(0, T, H^{3}\right)$ and there is $c>0$ such that

$$
\int_{0}^{T}\left|h_{x x x}\right|^{r / 5} d t \leq c \int_{0}^{T}\left(1+\left|h_{x}\right|^{r}\right) d t .
$$

REMARK 3.3. It is easy to check that the space $L^{r / 5}\left(0, T, H^{3}\right)$ is critical if and only if $L^{r}\left(0, T, H^{1}\right)$ is critical. Thus this result respects the criticality heuristic. In particular, the case $r \geq 8$ is covered by Theorem 3.1 above (for $\alpha=1$ ).

REMARK 3.4. If $h \in L^{\infty}\left(0, T, H^{1 / 2}\right)$ (critical) then by interpolation of $H^{1 / 2}$ and $H^{2}$ we obtain from the energy estimates that $h \in L^{6}\left(0, T, H^{1}\right)$, hence $h \in$ $L^{6 / 5}\left(0, T, H^{3}\right)$. So by interpolation of $H^{2}$ between $H^{1 / 2}$ and $H^{3}$ we recover $h \in$ $L^{2}\left(0, T, H^{2}\right)$. Thus this regularity result gives no improvement of the regularity given by the energy estimate in Section 1.2. It respects the level of criticality of the spaces.

Proof. Let $p=4-\frac{r}{2}$, then

$$
\frac{d}{d t} \frac{1}{\left(1+\left|h_{x}\right|_{L^{2}}^{2}\right)^{p}}=-2 p \frac{\left\langle h_{x t}, h_{x}\right\rangle_{L^{2}}}{\left(1+\left|h_{x}\right|_{L^{2}}^{2}\right)^{p+1}} .
$$

The fact that $h$ satisfies the PDE and integration by parts yield

$$
\left\langle h_{x t}, h_{x}\right\rangle_{L^{2}}=\frac{1}{2}\left|h_{x x}\right|_{L^{3}}^{3}-\left|h_{x x x}\right|_{L^{2}}^{2},
$$

while by the embedding of $H^{1 / 6}$ into $L^{3}$, interpolation and Young's inequality it follows that

$$
\left|h_{x x}\right|_{L^{3}}^{3} \leq C|h|_{13 / 6}^{3} \leq C\left|h_{x}\right|_{L^{2}}^{5 / 4}\left|h_{x x x}\right|_{L^{2}}^{7 / 4} \leq C\left|h_{x}\right|_{L^{2}}^{10}+\left|h_{x x x}\right|_{L^{2}}^{2} .
$$

Combining both results with the computation above yields

$$
\frac{\left|h_{x x x}\right|_{L^{2}}^{2}}{\left(1+\left|h_{x}\right|_{L^{2}}^{2}\right)^{p+1}} \leq c \frac{\left|h_{x}\right|_{L^{2}}^{10}}{\left(1+\left|h_{x}\right|_{L^{2}}^{2}\right)^{p+1}}+\frac{1}{p} \frac{d}{d t} \frac{1}{\left(1+\left|h_{x}\right|_{L^{2}}^{2}\right)^{p}} .
$$

Hence by the choice of $p$,

$$
\int_{0}^{T} \frac{\left|h_{x x x}\right|_{L^{2}}^{2}}{\left(1+\left|h_{x}\right|_{L^{2}}^{2}\right)^{p+1}} d t \leq c \int_{0}^{T}\left(1+\left|h_{x}\right|_{L^{2}}^{r}\right) d t
$$


By Hölder's inequality and the above estimate,

$$
\begin{aligned}
\int_{0}^{T}\left|h_{x x x}\right|_{L^{2}}^{\alpha} d t & \leq\left(\int_{0}^{T} \frac{\left|h_{x x x}\right|_{L^{2}}^{2}}{\left(1+\left|h_{x}\right|_{L^{2}}^{2}\right)^{p+1}} d t\right)^{\frac{\alpha}{2}}\left(\int_{0}^{T}\left(1+\left|h_{x}\right|_{L^{2}}^{2}\right)^{\frac{\alpha}{2-\alpha}(p+1)} d t\right)^{1-\frac{\alpha}{2}} \\
& \leq\left(\int_{0}^{T}\left(1+\left|h_{x}\right|_{L^{2}}^{r}\right) d t\right)^{\frac{\alpha}{2}}\left(\int_{0}^{T}\left(1+\left|h_{x}\right|_{L^{2}}^{2}\right)^{\frac{\alpha}{2-\alpha}(p+1)} d t\right)^{1-\frac{\alpha}{2}}
\end{aligned}
$$

and thus fixing the value $\alpha=\frac{r}{5}$ yields the claim.

3.4. Blow up below criticality. In this section we will study the blow up in a space below criticality, i.e. in some $H^{s}$ with $s<\frac{1}{2}$. This is a slight generalisation of Theorem 3.1 and prepares the results of Leray type shown later.

For $\frac{1}{4} \leq \delta \leq 1$ we obtain:

$$
\begin{aligned}
\frac{1}{2} \frac{d}{d t}|h|_{\delta}^{2} & \leq-c|h|_{2+\delta}^{2}+2 \int_{0}^{L}\left(-\partial_{x}^{2}\right)^{\delta} h_{x} \cdot h_{x} h_{x x} d x \\
& \leq-c|h|_{2+\delta}^{2}+C|h|_{1+2 \delta}|h|_{\frac{9}{4}}|h|_{\frac{5}{4}}
\end{aligned}
$$

where we have used the Sobolev embedding $H^{\frac{1}{4}} \subset L^{4}$.

REMARK 3.5. As it is used several times in the proofs, we state the following elementary interpolation inequality. For $\gamma>\alpha$ and $\beta \in[\alpha, \gamma]$,

$$
|h|_{\beta} \leq C|h|_{\alpha}^{\frac{\gamma-\beta}{\gamma-\alpha}}|h|_{\gamma}^{\frac{\beta-\alpha}{\gamma-\alpha}} .
$$

Fix $\gamma \in\left(\frac{1}{2}, \frac{5}{4}\right]$. If in formula (3.2) we use interpolation of $\dot{H}^{1+2 \delta}, \dot{H}^{5 / 4}$ and $\dot{H}^{9 / 4}$ between $H^{\gamma}$ and $H^{2+\delta}$, we get

$$
\frac{1}{2} \frac{d}{d t}|h|_{\delta}^{2} \leq-c|h|_{2+\delta}^{2}+C|h|_{\gamma}^{\frac{3+2 \delta}{4+2 \delta-2 \gamma}}|h|_{2+\delta}^{\frac{9+4 \delta-6 \gamma}{4+2 \delta-2 \gamma}}
$$

Using Young's inequality with exponents $p=(8+4 \delta-4 \gamma) /(9+4 \delta-6 \gamma)$ and $q=(8+4 \delta-4 \gamma) /(2 \gamma-1)$ we derive

$$
\frac{1}{2} \frac{d}{d t}|h|_{\delta}^{2} \leq C|h|_{\gamma^{\frac{2(3+2 \delta)}{2 \gamma-1}}} .
$$

This inequality implies the following result.

Theorem 3.6. Fix numbers $\gamma \in\left(\frac{1}{2}, \frac{5}{4}\right]$ and $\delta \in\left[\frac{1}{4}, 1\right]$ and let $h \in C^{\infty}\left(\left[0, t_{0}\right) \times\right.$ $[0, L])$ be a solution. If $|h(t)|_{\delta} \rightarrow \infty$ as $t \uparrow t_{0}$, then

$$
\int_{0}^{t_{0}}|h(t)|_{\gamma}^{\frac{2(3+2 \delta)}{2 \gamma-1}} d t=\infty
$$

Note that for a blow up below criticality with $\delta<\frac{1}{2}$ the $L^{p}\left([0, T], H^{\gamma}\right)$-norm in this theorem has a smaller $p$ than assured by Theorem 3.1. The spaces in the above theorem should always have the same level of criticality.

\section{Blow up}

In this section we discuss some properties of the blow up. First, at a possible blow-up time, one expects that all norms with higher regularity than the critical norm will blow up, in particular all $H^{s}$-norm with $s>1 / 2$ should blow up. In Subsection 4.2 we give a lower bound on the blow up in $H^{s}$-spaces, while in Subsection 4.4 we show a bound on the size of the set of singular times. We illustrate 
that a blow up to $-\infty$ is more likely, but first we give some remarks on possible shapes of a blow up.

4.1. Some remarks. Let us first give examples on which blow-up profiles $v=h(\tau)$ are possible at the blow-up time $\tau$.

- If $v$ exhibits a jump like $\operatorname{sign}(x)$, then the Fourier-coefficients decay like $1 / k$, and thus $v$ is in $H^{s}$ if and only if $s<\frac{1}{2}$.

- If $v$ exhibits a logarithmic pole like $\log (|x|)$, then the Fourier-coefficients decay like $1 / k$, and thus $v$ is in $H^{s}$ if and only if $s<\frac{1}{2}$.

- If $v$ exhibits a cusp like $|x|^{\alpha}$ for $\alpha \in(0,1)$, then the Fourier-coefficients decay like $|k|^{-(1+\alpha)}$, and thus $v$ is in $H^{1 / 2}$, and not a possible blow up.

4.1.1. Stationary solutions. The $L^{2}$ estimates (1.3) show that the only stationary solution is $h \equiv 0$, as $|h(t)|_{L^{2}} \rightarrow 0$ for $t \uparrow \infty$. On the other hand the problem is one-dimensional, so it is worth trying to look for solutions directly. The equation for stationary solutions is

$$
h_{x x x x}+\left(h_{x}^{2}\right)_{x x}=0,
$$

so there are constants $A, B$ such that $h_{x x}+h_{x}^{2}=A x+B$. By the periodic boundary conditions, $A=0$.

Case 1: $B=0$. By direct computations, we get

$$
h(x)=c_{1}+\log \left|1+c_{2} x\right|,
$$

and the only periodic solution corresponds to $c_{2}=0$, a constant function. Notice that, anyway, the solutions are singular with a log-like profile.

Case 2: $B=b^{2}$. Again by direct computations,

$$
h(x)=c_{1}+\log \left|\cosh b x+\frac{c_{2}}{b} \sinh b x\right|,
$$

and there are no periodic solutions. We remark that again the singularity has a log-like profile.

Case 3: $B=-b^{2}$. By elementary computations,

$$
h(x)=c_{1}+\log \left|b \cos b x+c_{2} \sin b x\right|,
$$

all solutions are periodic on $[0, L]$ as long as $b=\frac{2 \pi}{L} k$, for some $k \in \mathbf{N}$. If $x_{0}$ is any zero of $b \cos b x+c_{2} \sin b x$, we can write the solution as $\left.h(x)=c_{1}+\log \left|\sin b\left(x-x_{0}\right)\right|\right)$ (with a different value of $c_{1}$ ). Again, the stationary profile is log-like.

4.1.2. Self-similar solutions. By exploiting the scaling (2.1), we may look for solutions of the following kind,

$$
h(t, x)=\varphi\left(\frac{x}{\sqrt[4]{T-t}}\right),
$$

where $\varphi$ is a suitable function. The equation for $h$ reads in terms of $\varphi$ as

$$
\varphi_{y y y y}+\left(\varphi_{y}^{2}\right)_{y y}+y \varphi_{y}=0, \quad y \in \mathbf{R},
$$

and, by the regularity of weak solutions one shows easily that $\varphi, \varphi_{x x} \in L^{2}$ and hence $\varphi \in H^{2}(\mathbf{R})$. Here for simplicity we have neglected boundary conditions and formulated the problem on the whole real line. The problem above can be recast in weak form as

$$
\int \varphi \eta_{y y y y} d y+\int \varphi_{y}^{2} \eta_{y y} d y-\int \varphi \eta d y-\int y \varphi \eta_{y} d y=0, \quad \eta \in C_{c}^{\infty},
$$

where the solution $\varphi \in H_{\mathrm{loc}}^{1}(\mathbf{R})$. 
There is quite strong numerical evidence that there are no solutions to (4.1) defined on the whole $\mathbf{R}$. This fact would rule out self-similar solutions ${ }^{1}$.

4.2. Leray type results. We will prove the following theorem, which is based on one of the several celebrated results of Leray [14] on the Navier-Stokes equations. This relies mainly on a comparison result for ODEs (see Lemma B.1) and energy estimates. It improves the results of Theorem 3.1, which states that at blow up for $s>\frac{1}{2}$ the function $t \rightarrow|h(t)|_{s}^{8 /(2 s-1)}$ is not integrable. The result now says that it diverges at least as fast as $\frac{1}{t}$.

Theorem 4.1. Let $h \in C^{\infty}\left(\left[0, t_{0}\right) \times[0, L]\right)$ be a smooth local solution. Then for $s>\frac{1}{2}$ there is a universal constant $C>0$ such that $|h(t)|_{s} \rightarrow \infty$ for $t \uparrow t_{0}$ (or for any subsequence) implies

$$
|h(t)|_{s} \geq C\left(t_{0}-t\right)^{-(2 s-1) / 8} \quad \text { for all } t \in\left[0, t_{0}\right) .
$$

Proof. We proceed by using energy estimates. Again use the notation $D=$ $A^{1 / 4}=\left|\partial_{x}\right|$ and $B(u, v)=\left(u_{x} v_{x}\right)_{x x}$.

From (1.1) we obtain for $s=1+\delta$ with $\delta \in\left(-\frac{1}{2}, \frac{3}{2}\right)$

$$
\begin{aligned}
\frac{d}{d t}|h|_{1+\delta}^{2}+2|h|_{3+\delta}^{2} & =-2 \int_{0}^{L} D^{2 \delta} h_{x x} B(h, h) d x=4 \int_{0}^{L} D^{2 \delta} h_{x} B\left(h, h_{x}\right) d x \\
& \leq C|h|_{1+\delta+\epsilon}|h|_{\frac{5}{2}-\epsilon}|h|_{3+\delta},
\end{aligned}
$$

where we used Proposition A.4 with $\alpha=2+\delta, \beta=\frac{1}{2}-\epsilon$, and $\gamma=-\alpha+\epsilon$ for some small $\epsilon \in\left(0, \frac{1}{2}\right)$ such that $\epsilon+\delta \in\left(-\frac{1}{2}, \frac{3}{2}\right)$. Now interpolation (see Remark 3.5) yields

$$
\frac{d}{d t}|h|_{1+\delta}^{2}+2|h|_{3+\delta}^{2} \leq C|h|_{1+\delta}^{\frac{1}{4}(7-2 \delta)}|h|_{3+\delta}^{\frac{1}{4}(5+2 \delta)} .
$$

As $(5+2 \delta)<8$, we can apply Young's inequality with $p=8 /(7-2 \delta)$ and $q=$ $8 /(1+2 \delta)$ to derive

$$
\frac{d}{d t}|h|_{1+\delta}^{2} \leq C|h|_{1+\delta}^{2(5+2 \delta) /(1+2 \delta)}=C|h|_{s}^{2(3+2 s) /(2 s-1)} .
$$

Thus Lemma B.1 implies the theorem for $s \in\left(\frac{1}{2}, \frac{5}{2}\right)$.

Consider now $s=2+\delta$ with $\delta \in\left(-\frac{1}{2}, \frac{3}{2}\right)$.

$$
\begin{aligned}
\frac{d}{d t}|h|_{2+\delta}^{2}+2|h|_{4+\delta}^{2} & =2 \int_{0}^{L} D^{2 \delta} h_{x x x x} B(h, h) d x \\
& =-4 \int_{0}^{L} D^{2 \delta} h_{x x x} B\left(h, h_{x}\right) d x \\
& =-4 \int_{0}^{L} D^{2 \delta} h_{x x}\left[B\left(h_{x}, h_{x}\right)+B\left(h, h_{x x}\right)\right] d x \\
& \leq C|h|_{2+\delta+\epsilon}|h|_{\frac{7}{2}-\epsilon}|h|_{3+\delta}+C|h|_{3+\delta+\epsilon}|h|_{\frac{7}{2}-\epsilon}|h|_{2+\delta}
\end{aligned}
$$

\footnotetext{
${ }^{1}$ Existence of self-similar solutions has been a long standing problem for the NavierStokes equations. The problem was firstly posed by J. Leray [14] in 1934 and finally in 1996 Nečas, Růžička and Šverák [17] proved that there are no self-similar solutions with locally finite energy. Lately, Cannone and Planchon [9] proved existence of self-similar solution in Besov spaces.

Nečas et al. exploited a non-trivial maximum principle for $|u|^{2}+p$ (where $u$ is the velocity field and $p$ is the pressure). We remark that no such fact seems to be true in this case.
} 
where we again used Proposition A.4 with the same choice of $\alpha, \beta, \gamma$ and $\epsilon$. Now using interpolation,

$$
\frac{d}{d t}|h|_{2+\delta}^{2}+2|h|_{4+\delta}^{2} \leq C|h|_{2+\delta}^{\frac{1}{4}(7+2 \delta)}|h|_{4+\delta}^{\frac{1}{4}(5-2 \delta)},
$$

while by Young's inequality with $p=8 /(5-2 \delta)$ and $q=8 /(3+2 \delta)$ it follows that

$$
\frac{d}{d t}|h|_{2+\delta}^{2} \leq C|h|_{2+\delta}^{2(7+2 \delta) /(3+2 \delta)}=C|h|_{s}^{2(3+2 s) /(2 s-1)} .
$$

Now Lemma B.1 finishes the proof for $s \in\left(\frac{3}{2}, \frac{7}{2}\right)$.

The general case is proved similarly, by distributing the derivatives as evenly as possible on the tri-linear terms, as in the proof of Proposition 2.10, and then applying Proposition A.4, possibly with different $\alpha$ 's for different terms.

REMARK 4.2. We can also give a lower bound on the blow-up time $t_{0}$ depending on $|h(0)|_{\delta}$ for $\delta>\frac{1}{2}$. To be more precise, using the upper bound in Lemma B.1 the following is straightforward to verify. For all $s>\frac{1}{2}$ there is a constant $c_{s}>0$ such that the solution is regular and smooth on $\left(t, t_{*}\right)$ if $c_{s}|h(t)|_{s}^{8 /(2 s-1)}\left(t_{*}-t\right)<1$.

On the other hand, Theorem 4.1 immediately implies that near a blow up at $t_{*}$ we obtain for all $r \in\left(t, t_{*}\right)$, that $c_{s}|h(r)|_{s}^{8 /(2 s-1)}\left(t_{*}-r\right) \geq 1$.

4.3. Criterion for point-wise blow up to $-\infty$. We show that for a blow up in $L^{\infty}$ the blow up to $-\infty$ is much more likely than the blow up to $+\infty$. This is mainly based on the a-priori estimate from Section 1.3, but first we derive the following estimate:

$$
\begin{aligned}
\frac{1}{3} \frac{d}{d t} \int_{0}^{L} h^{3} d x & =-\int_{0}^{L} h^{2} h_{x x x x} d x-\int_{0}^{L} h^{2}\left(\left(h_{x}\right)^{2}\right)_{x x} d x \\
& =2 \int_{0}^{L} h h_{x} h_{x x x} d x+4 \int_{0}^{L} h\left(h_{x}\right)^{2} h_{x x} d x \\
& =-\int_{0}^{L} h\left(h_{x x}\right)^{2} d x-\frac{4}{3} \int_{0}^{L}\left(h_{x}\right)^{4} d x,
\end{aligned}
$$

where we used the cancellation property (1.2). Thus

$$
\int_{0}^{T} \int_{0}^{L}\left(h_{x}\right)^{4} d x d t \leq \int_{0}^{L} h^{3}(0) d x+\int_{0}^{T} \int_{0}^{L} h^{-}\left(h_{x x}\right)^{2} d x d t+\int_{0}^{L} h^{-} h^{2} d x .
$$

This implies a point-wise blow up to $-\infty$, as stated by the following theorem.

TheOREM 4.3. Let $h \in C^{\infty}([0, \tau) \times[0, L])$ be a smooth local solution. If $\int_{0}^{L} h^{3}(0) d x$ is finite and $\|h\|_{L^{4}\left(0, \tau, W^{1,4}\right)}=\infty$ then the negative part $h^{-}$has to blow up. In other words, there are $t_{n} \uparrow \tau$ and $x_{n} \in[0, L]$ such that $h\left(t_{n}, x_{n}\right) \rightarrow-\infty$.

CoROLLARY 4.4. If $\int_{0}^{L} h^{3}(0) d x<\infty$ and if $h^{-}$is uniformly bounded, then one obtains $\|h\|_{L^{4}\left(0, T, W^{1,4}\right)}<\infty$ and $\int_{0}^{T} \int_{0}^{L} h^{+}\left(h_{x x}\right)^{2} d x d t<\infty$.

Let us now show that not only we have a point-wise blow up, but also a blow up for some $\int_{0}^{L} \mathrm{e}^{-\gamma h(t)} d x$, while we know already by Section 1.3 that $\int_{0}^{L} \mathrm{e}^{\gamma h(t)} d x$ is finite for $\gamma \in(0,2)$. 
LEMMA 4.5. Let $h \in C^{\infty}([0, \tau) \times[0, L])$ be a smooth local solution. If

$$
\int_{0}^{t} \int_{0}^{L}\left|h_{x}\right|^{\alpha}|h|^{k} d x d t \longrightarrow \infty
$$

as $t \uparrow \tau$ for some $\alpha \in(0,4)$ and $k \geq 0$, then

$$
\int_{0}^{L} \mathrm{e}^{-\gamma h^{-}(t)} d x \longrightarrow \infty
$$

as $t \uparrow \tau$, for all $\gamma>0$.

PRoof. Applying twice Hölder's inequality and using (1.4) yield for any $\epsilon \epsilon$ $\left(0, \frac{\alpha}{2}\right)$ (that is, $\left.\frac{4 \epsilon}{\alpha} \in(0,2)\right)$,

$$
\begin{aligned}
\left(\int_{0}^{t} \int_{0}^{L}\right. & \left.\left|h_{x}\right|^{\alpha}|h|^{k} d x d t\right)^{\frac{4}{\alpha}} \leq \\
& \leq C \int_{0}^{t}\left(\int_{0}^{L}\left|h_{x}\right|^{\alpha}|h|^{k} d x\right)^{\frac{4}{\alpha}} d t \\
& \leq C \int_{0}^{t}\left(\int_{0}^{L} \mathrm{e}^{\frac{4 \epsilon}{\alpha}}\left|h_{x}\right|^{4} d x\right)\left(\int_{0}^{L} \mathrm{e}^{-\frac{4 \epsilon}{4-\alpha} h}|h|^{\frac{4 k}{4-\alpha}} d x\right)^{\frac{4-\alpha}{\alpha}} d t \\
& \leq C \sup _{[0, t]}\left(\int_{0}^{L} \mathrm{e}^{-\frac{4 \epsilon}{4-\alpha} h}|h|^{\frac{4 k}{4-\alpha}} d x\right)^{\frac{4-\alpha}{\alpha}} \int_{0}^{t} \int_{0}^{L} \mathrm{e}^{\frac{4 \epsilon}{\alpha} h}\left|h_{x}\right|^{4} d x d t \\
& \leq C(\alpha, h(0)) \sup _{[0, t]}\left(1+\int_{0}^{L} \mathrm{e}^{-\gamma h^{-}} d x\right)^{\frac{4-\alpha}{\alpha}},
\end{aligned}
$$

for $\gamma>\frac{4 \epsilon}{4-\alpha}$. Since $\epsilon$ can be chosen arbitrarily small, the lemma follows.

4.4. The set of singular times. Let $h$ be a weak solution to (1.1) and consider the set of regular times of $h$,

$\mathscr{R}=\left\{t \in(0, \infty): h\right.$ is continuous with values in $H^{1}$ in a neighbourhood of $\left.t\right\}$.

By Proposition 2.10, $\mathscr{R}$ is equal to the set of all times $t$ such that $h$ is $C^{\infty}$ in space and time in a neighbourhood of $t$. Define the set of singular times $\mathscr{S}=[0, \infty) \backslash \mathscr{R}$.

The next theorem proves (in the spirit of results of Leray [14], Scheffer [22] for Navier-Stokes), that the set of singular times is "small".

THEOREM 4.6. Given a weak solution $h$ to (1.1), the set $\mathscr{S}$ of singular times of $h$ is a compact subset of $[0, \infty)$ and

$$
\mathcal{H}^{\frac{1}{4}}(\mathscr{S})=0
$$

where $\mathcal{H}^{\frac{1}{4}}$ is the $\frac{1}{4}$-dimensional Hausdorff measure.

Proof. Fix a weak solution $h$ and define $\mathscr{R}$ and $\mathscr{S}$ as above. The proof is divided in four steps.

1. $\mathscr{S}$ is compact. The set $\mathscr{R}$ is clearly open, hence $\mathscr{S}$ is closed. We prove that $\mathscr{S}$ is bounded. Let $a_{\bullet}$ be the constant given in part $\mathbf{3}$ of Theorem 2.2. Assume by contradiction that $a_{\bullet}<|h(t)|_{\frac{1}{2}}$ for all $t \geq 0$. By interpolation and using the energy inequality (1.3),

$$
a_{\bullet}^{\frac{8}{3}} t<\int_{0}^{t}|h(s)|_{\frac{1}{2}}^{\frac{8}{3}} d s \leq \int_{0}^{t}|h(s)|_{L^{2}}^{\frac{2}{3}}|h(s)|_{2}^{2} d s \leq|h(0)|_{L^{2}}^{\frac{2}{3}} \int_{0}^{t}|h(s)|_{2}^{2} d s \leq 2|h(0)|_{L^{2}}^{\frac{8}{3}} .
$$


Hence for some $t_{0}>0,\left|h\left(t_{0}\right)\right|_{\frac{1}{2}} \leq a_{\bullet}$ and Theorems 2.2 and 2.11 imply that the solution $h$ is regular in $\left[t_{0}, \infty\right)$.

2. $\mathscr{S}$ has Lebesgue measure 0 . As any open set of $\mathbf{R}$ is the countable union of disjoint open intervals we have $\mathscr{R}=\bigcup_{j} I_{j}$, where the open intervals $I_{j}$ are the connected components of $\mathscr{R}$.

Define $\mathscr{S}_{2}=\left\{t: h(t) \notin \dot{H}^{2}\right\}$. Trivially, $\mathscr{R} \subset \mathscr{S}_{2}^{c}$, hence $\mathscr{S}_{2} \subset \mathscr{S}$. If $t_{0} \in$ $\mathscr{S} \backslash \mathscr{S}_{2}$, by Proposition $2.10 t_{0}$ is the endpoint of some $I_{j}$, hence $\mathscr{S} \backslash \mathscr{S}_{2}$ is at most countable. Finally, the energy estimate (1.3) implies that $\mathscr{S}_{2}$ has measure 0.

3. Estimate on the length of bounded $I_{j}$. Indeed, let $I_{j}$ be a bounded component of $\mathscr{R}$ and let $t_{1}, t_{2} \in I_{j}$. From Remark 4.2 we know $c\left(t_{2}-s\right)|h(s)|_{2}^{8 / 3} \geq 1$, and hence $c\left(t_{2}-s\right)^{-3 / 4} \leq|h(s)|_{2}^{2}$, for $s \in\left(t_{1}, t_{2}\right)$. Integrating for $s \in\left(t_{1}, t_{2}\right)$ and using the energy inequality (1.3), yields

$$
c\left(t_{2}-t_{1}\right)^{\frac{1}{4}} \leq \int_{t_{1}}^{t_{2}}|h(s)|_{2}^{2} d s \leq \frac{1}{2}|h(0)|_{L^{2}}^{2} .
$$

4. $\mathcal{H}^{\frac{1}{4}}(\mathscr{S})=0$. Write $I_{j}=\left(a_{j}, b_{j}\right)$ for bounded intervals. From (4.2) it follows that

$$
\sum_{j}\left(b_{j}-a_{j}\right)^{\frac{1}{4}}<\infty
$$

while $\sum_{j}\left(b_{j}-a_{j}\right)<\infty$, by the first step of the proof. Now we can proceed as in the proof of Theorem 2 of [22] to get the conclusion.

REMARK 4.7. It is possible to give a further limit to the set of singular times by showing that its box-counting dimension is smaller or equal than $\frac{1}{4}$. This result can be proved as in [21] and we refer to this paper for further details.

\section{Appendix A. An inequality for the non-linearity}

Given three real numbers $\alpha, \beta, \gamma$, consider the following condition.

Condition A.1. The real numbers $\alpha, \beta, \gamma$ satisfy

- $\alpha, \beta \geq 0$,

- $\alpha+\beta+\gamma \geq \frac{1}{2}$ with strict inequality if at least one is equal to $\frac{1}{2}$, and

- if $\gamma<0$, then either at least one of $\alpha$ and $\beta \leq \frac{1}{2}$, or at least one $\geq-\gamma$.

Lemma A.2. For every $\gamma \in \mathbf{R}$ there is $c>0$ such that for every $a \geq 1$,

$$
\sum_{|k| \leq a}|k|^{-2 \gamma} \leq \begin{cases}c a^{1-2 \gamma} & \gamma<\frac{1}{2} \\ c \log a & \gamma=\frac{1}{2}, \\ c & \gamma>\frac{1}{2} .\end{cases}
$$

Lemma A.3. Let $\alpha, \beta$ and $\gamma$ satisfy (A.1) (with $\alpha \leq \frac{1}{2}$ or $\beta \geq-\gamma$ when $\gamma<0$ ). Then there is $c>0$ such that for each $m \in \mathbf{Z}$, with $m \neq 0$,

$$
\sum_{\substack{|k|<2|m| \\ 0<|k-m|<\frac{1}{2}|k|}} \frac{1}{|k-m|^{2 \alpha}|k|^{2 \gamma}} \leq c|m|^{2 \beta} .
$$

Proof. Notice that, if $|k|<2|m|$ and $|k-m|<\frac{1}{2}|k|$, then $\frac{2}{3}|m| \leq|k|<2|m|$, since

$$
\frac{2}{3}|m| \leq \frac{2}{3}|k-m|+\frac{2}{3}|k|<\frac{1}{3}|k|+\frac{2}{3}|k|=|k| .
$$


Then apply Lemma A.2.

Consider

$$
B(u, v)=\left(u_{x} v_{x}\right)_{x x} .
$$

Proposition A.4. If $\alpha, \beta$ and $\gamma$ satisfy (A.1), there exists $c>0$ such that for all $u \in \dot{H}^{1+\alpha}, v \in \dot{H}^{1+\beta}$ and $w \in \dot{H}^{2+\gamma}$,

$$
\langle B(u, v), w\rangle \leq c|u|_{1+\alpha}|v|_{1+\beta}|w|_{2+\gamma} .
$$

Proof. Step 1. Write the functions $u, v, w$ in the Fourier expansion,

$$
u=\sum_{k \neq 0} u_{k} \mathrm{e}^{i k x}
$$

(and similarly for $v$ and $w$ ), so that

$$
\left(u_{x} v_{x}\right)_{x x}=\sum_{k \neq 0} k^{2}\left(\sum_{l+m=k} l m u_{l} v_{m}\right) \mathrm{e}^{i k x}
$$

and by the Cauchy-Schwarz inequality,

$$
\begin{aligned}
\langle B(u, v), w\rangle & =\sum_{k \neq 0} k^{2} \overline{w_{k}}\left(\sum_{l+m=k} l m u_{l} v_{m}\right) \\
& \leq|w|_{2+\gamma}\left[\sum_{k \neq 0}|k|^{-2 \gamma}\left(\sum_{l+m=k}\left|l m u_{l} v_{m}\right|\right)^{2}\right]^{\frac{1}{2}} .
\end{aligned}
$$

Hence, it is sufficient to analyse only the second term in the above product. Set for every $k \neq 0$,

$$
\begin{gathered}
A_{k}=\left\{(l, m): l+m=k,|l| \geq \frac{1}{2}|k|,|m| \geq \frac{1}{2}|k|\right\}, \\
B_{k}=\left\{(l, m): l+m=k,|l|<\frac{1}{2}|k|\right\}, \\
C_{k}=\left\{(l, m): l+m=k,|m|<\frac{1}{2}|k|\right\},
\end{gathered}
$$

and, for simplicity, $U_{l}=|l|^{1+\alpha}\left|u_{l}\right|$ and $V_{m}=|m|^{1+\beta}\left|v_{m}\right|$.

Step 2. We start by analysing the sum on $A_{k}$.

$$
\begin{aligned}
\sum_{k \neq 0}|k|^{-2 \gamma}\left(\sum_{A_{k}}\left|l m u_{l} v_{m}\right|\right)^{2} \leq & \sum_{k \neq 0}|k|^{-2 \gamma}\left(\sum_{A_{k}}|l|^{-\alpha}|m|^{-\beta} U_{l} V_{m}\right)^{2} \\
\text { (using Young's inequality) } \leq & c \sum_{k \neq 0}|k|^{-2 \gamma}\left(\sum_{A_{k}}|l|^{-\alpha-\beta} U_{l} V_{m}\right)^{2} \\
& +c \sum_{k \neq 0}|k|^{-2 \gamma}\left(\sum_{A_{k}}|m|^{-\alpha-\beta} U_{l} V_{m}\right)^{2}
\end{aligned}
$$

the two terms are similar, we bound only the first one,

$$
\begin{gathered}
\text { (using the Cauchy-Schwarz inequality) } \leq c \sum_{k \neq 0}|k|^{-2 \gamma}\left(\sum_{A_{k}}|l|^{-2(\alpha-\beta)} U_{l}^{2}\right)\left(\sum_{A_{k}} V_{m}^{2}\right) \\
\text { (switching the sums) } \leq c|v|_{1+\beta}^{2} \sum_{l \neq 0}|l|^{-2(\alpha+\beta)} U_{l}^{2}\left(\sum_{|k| \leq 2|l|}|k|^{-2 \gamma}\right) \\
\text { (using Lemma A.2) } \leq c|u|_{1+\alpha}^{2}|v|_{1+\beta}^{2} .
\end{gathered}
$$


Step 3. Next, we analyse the sum on $B_{k}$ (the sum on $C_{k}$ being entirely similar). Notice that, when using the Cauchy-Schwarz inequality below, we are free to weigh either the terms in $u$ or in $v$ with derivatives. We shall choose one of the two depending on the values of $\gamma$ (wherever we need an exponent to be $\leq \frac{1}{2}$ or $\geq-\gamma$, according to condition (A.1)).

$$
\begin{aligned}
\sum_{k \neq 0}|k|^{-2 \gamma}\left(\sum_{B_{k}}\left|l m u_{l} v_{m}\right|\right)^{2} & \leq \sum_{k \neq 0}|k|^{-2 \gamma}\left(\sum_{B_{k}}|l|^{-\alpha}|m|^{-\beta} U_{l} V_{m}\right)^{2} \\
\text { (Cauchy-Schwarz' inequality) } & \leq \sum_{k \neq 0}|k|^{-2 \gamma}\left(\sum_{B_{k}}|l|^{-2 \alpha}|m|^{-2 \beta} V_{m}^{2}\right)\left(\sum_{B_{k}} U_{l}^{2}\right) \\
\text { (switching the sums) } & \leq|u|_{1+\alpha}^{2} \sum_{m \neq 0}|m|^{-2 \beta} V_{m}^{2}\left(\sum_{\substack{|k|<2|m| \\
0<|k-m|<\frac{1}{2}|k|}}|k-m|^{-2 \alpha}|k|^{-2 \gamma}\right) \\
\text { (using Lemma A.3) } & \leq c|u|_{1+\alpha}^{2}|v|_{1+\beta}^{2} .
\end{aligned}
$$

The proof is complete.

\section{Appendix B. Blow up for ODEs}

The following elementary lemma is crucial to prove Leray type bounds. We state and prove it for completeness.

LEMMA B.1. Let $\varphi:\left(0, t_{0}\right) \rightarrow \mathbb{R}$ be a non-negative function such that for $p>1$ we have $\dot{\varphi} \leq C \varphi^{p}$, on $\left(0, t_{0}\right)$. Then, $\varphi\left(t_{n}\right) \uparrow \infty$ for a subsequence $t_{n} \uparrow t_{0}$, implies

$$
\varphi(t) \geq\left[(p-1) C\left(t_{0}-t\right)\right]^{-1 /(p-1)} \quad \text { for all } t \in\left(0, t_{0}\right) \text {. }
$$

Moreover,

$$
\varphi(t) \leq\left[\varphi(s)^{-(p-1)}+C(p-1) s-C(p-1) t\right]^{-\frac{1}{p-1}} \quad \text { for all } 0<s<t<t_{0}
$$

ProOF. We have for $t>s$

$$
\frac{1}{p-1}\left(\varphi(s)^{-(p-1)}-\varphi(t)^{-(p-1)}\right)=\int_{\varphi(s)}^{\varphi(t)} \frac{1}{z^{p}} d z=\int_{s}^{t} \frac{\dot{\varphi}}{\varphi^{p}} d \tau \leq C(t-s)
$$

Now for $t_{n} \uparrow t_{0}$ we obtain

$$
\frac{1}{p-1} \varphi(t)^{-(p-1)} \leq C\left(t_{0}-t\right)
$$

and finally

$$
\varphi(t) \geq\left[(p-1) C\left(t_{0}-t\right)\right]^{-1 /(p-1)}
$$

for all $t \in\left(0, t_{0}\right)$.

For the second result

$$
\varphi(s)^{-(p-1)}-C(p-1)(t-s) \leq \varphi(t)^{-(p-1)}
$$

and thus

$$
\varphi(t) \leq\left[\varphi(s)^{-(p-1)}+C(p-1) s-C(p-1) t\right]^{-1 /(p-1)}
$$




\section{Appendix C. Analytic Semigroups}

The following properties of analytic semigroups are well known, but we give short sketches of proofs for the sake of completeness.

Lemma C.1. Consider $A=\partial_{x}^{4}$ subject to periodic boundary conditions on $[0, L]$ and $T>0$. For all $u=\sum_{k} u_{k} e_{k} \in L^{2}$ and $\alpha>0$,

$$
\left|s^{\alpha} A^{\alpha} \mathrm{e}^{-s A} u\right|_{L^{2}} \rightarrow 0, \quad \text { for } s \rightarrow 0,
$$

and for all $f \in L^{\infty}\left(0, T, L^{2}\right)$, with 0 average on $(0,1)$, and $1+a-b=0$, with $a>-1$ and $b<1$,

$$
I_{a, b}(f)(t)=\int_{0}^{t} s^{a} A^{b} \mathrm{e}^{-(t-s) A} f(s) d s
$$

converges to 0 in $L^{2}$ as $t \rightarrow 0$.

Proof. The first statement is obvious by Lebesgue theorem, since

$$
\left|s^{\alpha} A^{\alpha} \mathrm{e}^{-s A} u\right|_{L^{2}}^{2} \leq C \sum_{k}\left(k^{4} s\right)^{2 \alpha} \mathrm{e}^{-c s k^{4}} u_{k}^{2}
$$

For the second statement note that (with a change of variables) $\left|I_{a, b}(f)(t)\right|_{L^{2}}^{2}$ is equal to

$$
\begin{aligned}
& \sum_{k \neq 0} k^{8 b} \int_{0}^{t} \int_{0}^{t} s^{a} r^{a} k^{8 b} \mathrm{e}^{-c(2 t-s-r) k^{4}} f_{k}(s) f_{k}(r) d d s d r= \\
& =t^{2 a+2} \int_{0}^{1} \int_{0}^{1}(1-s)^{a}(1-r)^{a} \sum_{k \neq 0} k^{8 b} \mathrm{e}^{-c(s+r) t k^{4}} f_{k}(t-t s) f_{k}(t-t r) d s d r \\
& =t^{2 b} \int_{0}^{1} \int_{0}^{1}(1-s)^{a}(1-r)^{a}\left[\left(\sum_{(s+r) t k^{4} \leq \epsilon}\right.\right. \\
& \left.\left.+\sum_{(s+r) t k^{4}>\epsilon}\right) k^{8 b} \mathrm{e}^{-c(s+r) t k^{4}} f_{k}(t-t s) f_{k}(t-t r)\right] d s d r \\
& \leq c \int_{0}^{1} \int_{0}^{1} \frac{(1-s)^{a}(1-r)^{a}}{(s+r)^{2 b}}\left(\epsilon^{2 b}\|f\|_{L^{\infty}\left(L^{2}\right)}+\sum_{(s+r) t k^{4}>\epsilon} f_{k}(t-t s) f_{k}(t-t r)\right) d s d r,
\end{aligned}
$$

which goes to zero by Lebesgue theorem if one first takes the limit as $t \rightarrow 0$ and then as $\epsilon \downarrow 0$, since the function $(1-r)^{a}(1-s)^{a}(r+s)^{-2 b}$ is integrable and the other term is bounded for $\epsilon \leq 1$ and $t \leq T$.

\section{Acknowledgements}

The authors are grateful to the two referees and especially to the editor James Robinson for many helpful comments that improved the paper.

\section{References}

[1] A. L. Barabasi, H. E. Stanley, Fractal concepts in surface growth, Cambridge University Press, 1995.

[2] J. T. Beale, T. Kato, A. Majda, Remarks on the breakdown of smooth solutions for the 3-D Euler equations, Comm. Math. Phys. 94, no. 1 (1984), 61-66.

[3] D. Blömker, M. Hairer, Stationary solutions for a model of amorphous thin-film growth, Stochastic Anal. Appl. 22 (2004), no. 4, 903-922. 
[4] D. Blömker, C. GugG, On the existence of solutions for amorphous molecular beam epitaxy, Nonlinear Anal. Real World Appl. 3 (2002), no. 1, 61-73.

[5] D. Blömker, C. Gugg, M. Raible, Thin-film-growth models: roughness and correlation functions, European J. Appl. Math. 13 (2002), no. 4, 385-402.

[6] D. Blömker, C. GugG, Thin film growth models: On local solutions, Recent developments in stochastic analysis and related topics, World Scientific, Singapore. Proceedings of the first Sino-German conference on stochastic analysis, S. Albeverio, Z. M. Ma, M. Röckner (2004), 66-77.

[7] D. Blömker, F. Flandoli, M. Romito, Markovianity and ergodicity for a surface growth PDE, Annals of Probability, 37 (2009), no. 1, 275-313.

[8] L. Caffarelli, R. Kohn, L. Nirenberg, Partial regularity of suitable weak solutions of the Navier-Stokes equations, Comm. Pure Appl. Math. 35 (1982), no. 6, 771-831.

[9] M. Cannone, F. Planchon, Self-similar solutions for Navier-Stokes equations in $R^{3}$, Comm. PDE 21 (1996), no. 1-2, 179-193.

[10] M. Cannone, Harmonic analysis tools for solving the incompressible Navier-Stokes equations, in Handbook of Mathematical Fluid Dynamics, vol. 3, S. Friedlander and D. Serre eds., 161244, North-Holland, Amsterdam, 2004.

[11] H. Fujita, T. Kato, On the Navier-Stokes initial value problem. I, Arch. Rational Mech. Anal. 16 (1964), 269-315.

[12] T. Halpin-Healy, Y. C. Zhang, Kinetic roughening phenomena, stochastic growth, directed polymers and all that, Physics Reports, 254 (1995), 215-414.

[13] H. Koch, D. Tataru, Well-posedness for the Navier-Stokes equations, Adv. Math. 157 (2001), no. $1,22-35$.

[14] J. LeRAY, Sur le mouvement d'un liquide visqueux emplissant l'espace, Acta Math. 63 (1934), no. $1,193-248$.

[15] D. Li, YA. G. SinaI, Blow ups of complex solutions of the 3D Navier-Stokes system and renormalization group method, J. Eur. Math. Soc. 10 (2008), no. 2, 267-313.

[16] D. LI, YA. G. SinaI, Complex singularities of solutions of some $1 D$ hydrodynamic models, Physica D 237 (2008), 1945-1950.

[17] J. NeČAs, M. RŮŽIČKA, V. Šverák, On Leray's self-similar solutions of the Navier-Stokes equations, Acta Math. 176 (1996), no. 2, 283-294.

[18] G. Prodi, Un teorema di unicit per le equazioni di Navier-Stokes (Italian), Ann. Mat. Pura Appl. 48 (1959), no. 4, 173-182.

[19] M. Raible, S. G. Mayr, S. J. Linz, M. Moske, P. Hänggi, K. Samwer, Amorphous thin film growth: Theory compared with experiment, Europhysics Letters, 50 (2000), 61-67.

[20] M. Raible, S. J. Linz, P. HäNGGi, Amorphous thin film growth: Minimal deposition equation, Physical Review E, 62 (2000), 1691-1705.

[21] J. C. Robinson, W. Sadowski, Decay of weak solutions and the singular set of the threedimensional Navier-Stokes equations, Nonlinearity, 20 (2007), no. 5, 1185-1191.

[22] V. Scheffer, Turbulence and Hausdorff dimension, (Proc. Conf., Univ. Paris-Sud, Orsay, 1975), pp. 174-183. Lecture Notes in Math. 565, Springer, Berlin, 1976.

[23] J. SERRIN, On the interior regularity of weak solutions of the Navier-Stokes equations, Arch. Rational Mech. Anal. 9 (1962), 187-195.

[24] M. Siegert, M. Plischke, Solid-on-solid models of molecular-beam epitaxy, Physical Review E, 50 (1994), 917-931.

[25] O. Stein, M. Winkler, Amorphous molecular beam epitaxy: global solutions and absorbing sets, European J. Appl. Math. 16 (2005), no. 6, 767-798.

Institut für Mathematik, Universität Augsburg, D-86135 Augsburg, Germany

E-mail address: dirk.bloemker@math.uni-augsburg.de

Dipartimento di Matematica, Università di Firenze, Viale Morgagni 67/A, I-50134

Firenze, Italia

E-mail address: romito@math.unifi.it 